\title{
Coding Scheme Optimization for Fast Fluorescence Lifetime Imaging
}

\author{
JONGHO LEE, JENU VARGHESE CHACKO, BING DAI, SYED AZER REZA, ABDUL KADER SAGAR, \\ KEVIN W. ELICEIRI, ANDREAS VELTEN, and MOHIT GUPTA, University of Wisconsin-Madison
}

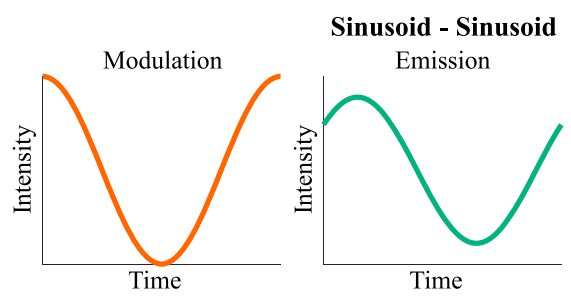

(a) Conventional coding scheme

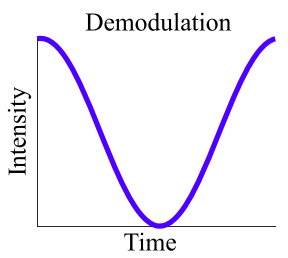

Time

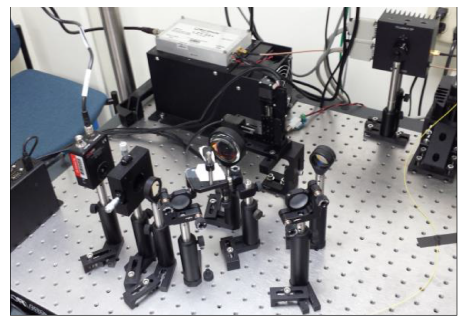

(c) Hardware prototype

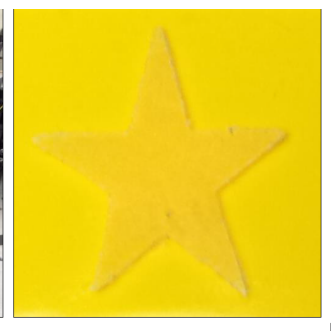

(d) Fluorescent sample

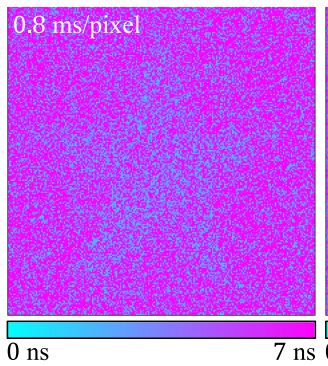

(e) Result with short acquisition time using (a)

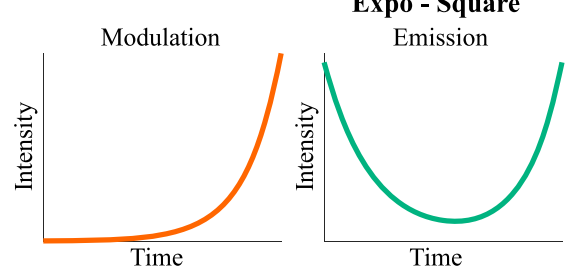

(b) Our coding scheme
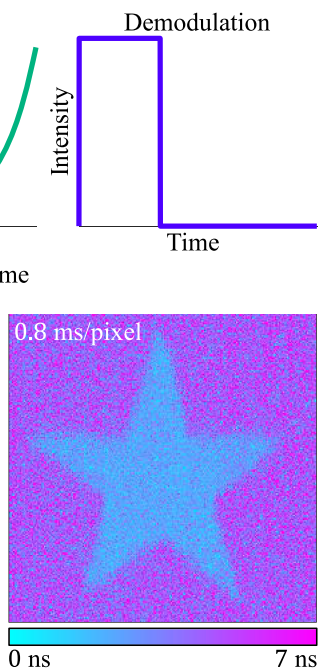

(g) Result with short acquisition time using (b)

Fig. 1. Fast frequency domain fluorescence lifetime imaging (FLIM). (a)-(b) Fluorescence excitations by different modulation functions (orange) lead to different fluorescence emissions (green). The intensities obtained by correlating the emissions with demodulation functions (blue) determine the SNR. We propose a theoretical framework for analysis and design of FD-FLIM coding schemes (modulation and demodulation functions), and use that to design (b) novel coding schemes that achieve considerably higher SNR as compared to conventional methods. (c) We developed a prototype FD-FLIM system to implement various coding schemes. (d) A fluorescent sample with two different fluorescence lifetimes for the foreground and the background is excited by a low power light source. (e) With the conventional coding scheme and $0.8 \mathrm{~ms} /$ pixel acquisition time, no clear boundary is observed between the foreground and background. (f) A considerably longer (10ms/pixel) acquisition time is required to obtain a clear boundary. (g) With the proposed coding schemes, $0.8 \mathrm{~ms} /$ pixel acquisition time is sufficient to detect a clear boundary.

Fluorescence lifetime imaging (FLIM) is used for measuring material properties in a wide range of applications, including biology, medical imaging, chemistry, and material science. In frequency-domain FLIM (FD-FLIM), the object of interest is illuminated with a temporally modulated light

This work is supported in part by the DARPA REVEAL program DARPA0, by the ONR ONR0 under Grants No. N000141612995 and No. N000141512652I ONR0, and by the NIH NIH0 under Grant No. R01 CA185251 NIH0.

Authors' addresses: J. Lee and M. Gupta, 1210 W. Dayton Street, Department of Computer Sciences, UW-Madison, Madison, WI 53706; emails: \{jongho, mohitg\}@cs.wisc.edu; J. V. Chacko, B. Dai, A. K. Sagar, and K. W Eliceiri, 1675 Observatory Drive, Laboratory for Optical and Computational Instrumentation, UW-Madison, Madison, WI 53706; emails: \{jenu.chacko, bdai6, msagar, eliceiri\}@wisc.edu; S. A. Reza and A. Velten, 1300 University Ave., Medical Sciences Building, UW-Madison, Madison, WI 53706; emails: \{sreza2, velten\}@wisc.edu.

Permission to make digital or hard copies of all or part of this work for personal or classroom use is granted without fee provided that copies are not made or distributed for profit or commercial advantage and that copies bear this notice and the full citation on the first page. Copyrights for components of this work owned by others than ACM must be honored. Abstracting with credit is permitted. To copy otherwise, or republish, to post on servers or to redistribute to lists, requires prior specific permission and/or a fee. Request permissions from permissions@acm.org.

(C) 2019 Association for Computing Machinery.

0730-0301/2019/06-ART26 \$15.00

https://doi.org/10.1145/3325136 source. The fluorescence lifetime is measured by computing the correlations of the emitted light with a demodulation function at the sensor. The signal-to-noise ratio (SNR) and the acquisition time of a FD-FLIM system is determined by the coding scheme (modulation and demodulation functions). In this article, we develop theory and algorithms for designing highperformance FD-FLIM coding schemes that can achieve high SNR and short acquisition time, given a fixed source power budget. Based on a geometric analysis of the image formation and noise model, we propose a novel surrogate objective for the performance of a given coding scheme. The surrogate objective is extremely fast to compute, and can be used to efficiently explore the entire space of coding schemes. Based on this objective, we design novel, high-performance coding schemes that achieve up to an order of magnitude shorter acquisition time as compared to existing approaches. We demonstrate the performance advantage of the proposed schemes in a variety of imaging conditions, using a modular hardware prototype that can implement various coding schemes.

CCS Concepts: • Computing methodologies $\rightarrow$ Computational photography;

Additional Key Words and Phrases: Fluorescence lifetime, coding optimization, waveform optimization, time-of-flight 


\section{ACM Reference format:}

Jongho Lee, Jenu Varghese Chacko, Bing Dai, Syed Azer Reza, Abdul Kader Sagar, Kevin W. Eliceiri, Andreas Velten, and Mohit Gupta. 2019. Coding Scheme Optimization for Fast Fluorescence Lifetime Imaging. ACM Trans. Graph. 38, 3, Article 26 (June 2019), 16 pages.

https://doi.org/10.1145/3325136

\section{INTRODUCTION}

Fluorescence is the phenomena where a material absorbs light at certain wavelengths, and then emits light at different, typically longer wavelengths. The wavelengths at which light is absorbed and emitted are characteristic of the physical and chemical properties of the material (Hollas 2004). Therefore, the excitation and emission spectra are used as signatures for material recognition in a variety of applications, such as wine classification (AiradoRodríguez et al. 2009), honey classification (Lenhardt et al. 2015), and oil classification (Sikorska et al. 2005).

Spectrum based fluorescence imaging techniques rely on steady-state measurements, i.e., the measurements are assumed to be constant over time. However, fluorescence also manifests as a transient (high-speed temporal) phenomena. When the molecule of a fluorescent material absorbs a photon, it goes into an excited state. The molecule returns to the ground state after a time delay, when it emits a photon. The delay between absorption and emission of photon is a random variable (due to the stochastic nature of emission), with an exponential distribution (Becker 2014). The mean delay, i.e., the average time the molecule spends in the excited state prior to returning to the ground state, is called fluorescence lifetime. The typical scale of the fluorescent lifetime is from several picoseconds (ps) to several nanoseconds (ns) (Bastiaens and Squire 1999).

Due to the exponential distribution of time delay, if a fluorescent material is illuminated with a short (e.g., few picoseconds duration) light pulse, the intensity of the emitted light decays exponentially over time. The rate of the exponential decay and the fluorescence lifetime provide information about material properties (e.g., temperature, viscosity, $\mathrm{pH}$ (Shi et al. 2014), chemical concentration (Sun et al. 2015)) that is often not available in the steady-state fluorescence spectral measurements (Berezin and Achilefu 2010). As a result, fluorescence lifetime imaging (FLIM) has found applications in several domains, including biology, medicine, chemistry, material science, agriculture, and art, where it has been used to distinguish between cancerous and noncancerous tissue (Colasanti et al. 2000; Pradhan et al. 1995), perform fingerprint detection (Seah et al. 2005, 2006), non-destructive fruit quality detection (Kim et al. 2008), non-invasive artwork analysis (Comelli et al. 2004; Nevin et al. 2007), and wood classification (Donaldson and Radotic 2013).

Broadly, FLIM techniques can be classified two ways: time domain (TD) FLIM, and frequency domain (FD) FLIM. In TD-FLIM, the sample of interest is illuminated with a short light pulse. A high-speed sensor (e.g., a single photon avalanche diode (Pancheri et al. 2013)) records the time profile of the emitted light, from which lifetime is extracted by performing an exponential function fit. Although TD-FLIM achieves high precision, it requires expensive components (short-pulse laser, high-speed sensor) (Elson et al. 2004). Furthermore, since the sensor requires capturing the entire time profile of the emitted light, in most cases (e.g., TD-FLIM by time-correlated single photon counting), the sample needs to be scanned one pixel at a time due to bandwidth constraints, making it prohibitively slow, especially for in vivo diagnostic applications where the motion artifacts need to be minimized (Requejo-Isidro et al. 2004).

Our focus is on frequency domain FLIM (FD-FLIM), where the sample is illuminated continuously (instead of with short pulses), with a light source whose brightness is modulated over time. The sensor captures intensity measurements by correlating the emitted light with a demodulation function, over relatively long integration times (e.g., few milliseconds). FD-FLIM requires capturing a small number (as few as three) of correlation intensity measurements in order to recover the lifetime (Elder et al. 2008), with relatively low cost components. The signal-to-noise ratio (SNR), and hence, the acquisition time, of a FD-FLIM system is determined by the coding scheme (modulation and demodulation functions) used. Most existing FD-FLIM systems use sinusoid (Philip and Carlsson 2003) or square waves (Booth and Wilson 2004; Schlachter et al. 2009). For example, an often used FD-FLIM coding scheme is sinusoid coding, where both modulation and demodulation functions are sinusoids of the same frequency (Philip and Carlsson 2003). Unfortunately, the SNR achieved by sinusoid coding remains low, thus often requiring long acquisition times to achieve the desired precision.

We propose a geometric framework for design and analysis of novel, high-performance FD-FLIM coding schemes. We define the mean lifetime error, a measure of coding scheme performance, based on a geometric analysis of the FD-FLIM image formation model. The mean lifetime error is conceptually easy to understand, and can be used to predict the performance of a given coding scheme over a specified range of lifetimes. However, mean lifetime error requires expensive numerical computations. Therefore, a coding scheme optimization procedure that directly uses the mean lifetime error as an objective function, remains prohibitively expensive from a computational standpoint.

Contributions and Implications. Our main theoretical contribution is the derivation of a computationally lightweight surrogate for the mean lifetime error based on the first-order partial derivative of the FD-FLIM image formation equation. The surrogate is considerably faster to compute than the mean lifetime error, and thus, enables us to efficiently explore the large space of FD-FLIM coding functions to find the optimal solution. Based on this surrogate objective, we design FD-FLIM coding schemes that achieve up to an order of magnitude higher acquisition speed (given a fixed source power budget), as compared to conventional schemes. An example is shown in Figure 1. FD-FLIM can be considered a special case (with an exponential impulse scene response) of transient imaging. Due to this relationship, the scope of transient and time-of-flight (ToF) imaging principles developed in graphics and computer vision could be expanded to a broader set of applications involving FLIM (e.g., medical and clinical). Furthermore, the proposed FD-FLIM measurement optimization approaches can be used in transient imaging scenarios where the scene's impulse response can be modeled as an exponential function (e.g., single scattering (Wu et al. 2012)). 
Hardware Prototype. We develop a proof-of-concept hardware prototype (Figure 1(c)) capable of implementing a wide range of coding schemes, and demonstrate the performance benefit of the proposed coding schemes, for several materials and a broad range of SNR scenarios. Our current hardware prototype is slow in acquisition because it is based on mechanical scanning. The proposed coding schemes can potentially be implemented on fullframe FLIM systems (Requejo-Isidro et al. 2004) for real-time FLIM measurements (Bhandari et al. 2015a).

\section{RELATED WORK}

Performance Metric and Code Design for FLIM. There has been surprisingly little work on optimization of coding functions for FLIM. While a few specific functions (e.g., sinusoid, square waves, impulse function) have been evaluated in terms of a figure of merit called F-value (Elder et al. 2008; Esposito et al. 2007; Lin and Gmitro 2010; Philip and Carlsson 2003), there is no general recipe for designing optimal FD-FLIM coding schemes. Our goal is to develop universal, easy to compute, performance metrics for the entire space of coding schemes, which can be used to design and analyze novel, high-performance coding schemes that considerably outperform existing approaches in a broad range of scenarios.

Fluorescence in Computer Vision and Graphics: Fluorescence-based phenomena have been used in computer vision and graphics in various contexts. For example, the observation that fluorescent materials behave approximately like Lambertian surfaces (emits light equally in all directions) was used to reconstruct 3D shape (Sato et al. 2012; Treibitz et al. 2012). Hullin et al. (2008) developed a technique to reconstruct 3D shape of transparent objects by immersing them in fluorescent liquid. Fu et al. (2014) proposed an inter-reflection removal method using reflective and fluorescent components in two channels from the captured image of a fluorescent object. Han et al. (2012) used fluorescence emission for estimating camera spectral sensitivity. Approaches for jointly capturing (Hullin et al. 2010), and using reflectance and fluorescent emission images, e.g., for improved classification of coral reef images (Beijbom et al. 2016), have also been proposed. These approaches are based on steady-state fluorescence spectral measurements. In contrast, this article focuses on the transient aspects of fluorescence.

Relationship to Transient Imaging. The goal of transient imaging is to recover the entire time profile of light transport. $\mathrm{Wu}$ et al. (2012) decomposed light transport into individual components (direct, indirect, scattering, inter-reflections) for scene analysis applications. Velten et al. (2012) recovered 3D shape around a corner using transient imaging. Heide et al. (2013, 2014) obtained transient images by a correlation-based ToF image sensor.

In general, the structure of transient waveforms depends on scene complexity, and can have arbitrary shapes. Typically, hundreds of measurements, per-pixel, are required to recover the transient waveforms. In contrast, the focus of this article is on FLIM, where the incident waveform can be modeled by an exponentially decaying function with a single parameter (Equation (1)). Therefore, only a few measurements are sufficient to recover the fluorescence lifetime. The proposed approaches can be relevant in transient imaging scenarios where the scene's impulse response can be modeled as a low-dimensional parametric function. For example, subsurface scattering, among various light transport components, can be modeled with an exponentially decaying function where the decay parameter is related with concentration of scattering media (Wu et al. 2012).

Fluorescence Lifetime Estimation with ToF sensors. Recently, approaches based on ToF sensors have been proposed for low-cost fluorescence lifetime estimation. Esposito et al. showed that $\mathrm{CCD} / \mathrm{CMOS}$ sensors, originally developed for depth sensing, can be used for lifetime estimation (Esposito et al. 2006, 2005). Bhandari et al. also showed that lifetime can be estimated with ToF sensors using codes unifying time domain and and frequency domain approaches (Bhandari et al. 2015b; Bhandari and Raskar 2016). Our goal is different. Instead of analyzing specific hardware implementations and sensors, we develop a general coding theory for FD-FLIM. We envision that the proposed coding schemes and optimization approaches, along with modern transient and ToF sensors, will enable faster and low-cost hardware implementation of FD-FLIM in the future.

\section{IMAGE FORMATION MODEL}

\subsection{Fluorescence Transient Response}

The fluorescence transient response $h(t ; \tau)$ of a material is defined as the exponentially decaying temporal intensity profile of the emitted light, after the material is illuminated with a light impulse $\delta(t) . h(t ; \tau)$ is given as

$$
h(t ; \tau)=\frac{1}{\tau} e^{-\frac{t}{\tau}} \quad(t \geq 0),
$$

where $\tau$ is the material's fluorescence lifetime; it determines the rate of exponential decay. ${ }^{1} h(t ; \tau)$ is normalized so that $\int_{0}^{\infty} h(t ; \tau)=1$.

\subsection{FD-FLIM Imaging Model}

A FD-FLIM imaging system consists of a light source (e.g., a laser diode) used to illuminate the sample of interest, and a sensor used to capture the fluorescence emission, as shown in Figure 2. Let the intensity of the source be temporally modulated according to a periodic function $M(t)(M(t) \geq 0)$, also called the modulation or the excitation function. Due to absorption of incident light and fluorescence, the sample emits light, which is captured by the sensor. Then, the fluorescence emission $E(t),{ }^{2}$ as observed at the sensor, is given as a scaled and offset version of the convolution of $M(t)$

\footnotetext{
${ }^{1}$ We assume mono-exponential decay, where the fluorescence emission intensity follows a single exponential function. Certain fluorescent materials have multiexponential decay, where the fluorescence emission profile is a linear combination of multiple exponential functions (Lakowicz 2006). While designing coding schemes for multi-exponential decay is outside the scope of this article, the approaches presented in this article can potentially be extended for multi-exponential lifetime decay estimation. This forms a promising future research direction.

${ }^{2}$ The wavelengths of the emitted light $E(t)$ and incident signal $M(t)$ are different due to fluorescence spectral shift (also called Stokes shift). In this article, for ease of notation, we ignore the wavelength dependence, and consider only the temporal variation of the incident and emitted signals. In practice, a dichroic mirror can be used to reflect the incident light to the sample and to transmit the fluorescence emission to the sensor, based on the wavelength, as shown in Figure 2.
} 
Function generator

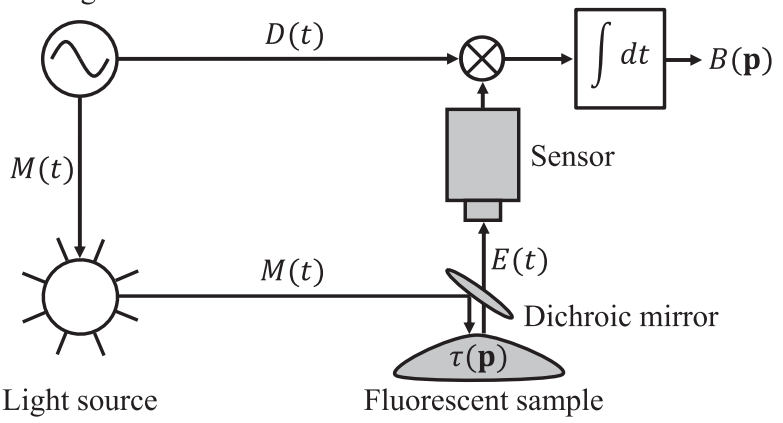

Fig. 2. Image formation model of FD-FLIM. A light source with temporally modulated intensity according to function $M(t)$ excites a fluorescent sample. The resulting fluorescence emission $E(t)$ captured by a sensor is correlated with the demodulation function $D(t)$ to get the image intensity $B$. The lifetime $\tau$ is measured from an intensity vector $\mathbf{B}=\left[B_{1}, B_{2}, \ldots, B_{K}\right](K \geq 3)$ where each measurement $B_{i}$ is captured using a different pair of modulation and demodulation functions.

and $h(t ; \tau)$ :

$$
E(t ; \tau)=\alpha(M \otimes h)(t ; \tau)+\gamma=\alpha \int_{-\infty}^{\infty} M(s ; \tau) h(t-s ; \tau) d s+\gamma,
$$

where $\alpha$ is a scale factor depending on system parameters (e.g., system instrumentation) and the sample (e.g., concentration, quantum yield). $\gamma$ is an offset corresponding to ambient illumination due to external sources and $\otimes$ is a convolution operator. Equation (2) does not account for the temporal shift in $E(t)$ caused by the travel time along the excitation path (light source $\rightarrow$ sample) and the emission path (sample $\rightarrow$ sensor). We assume this shift can be computed independently and compensated by a calibration process.

The sensor computes the correlation of the emitted light $E(t ; \tau)$ with a demodulation function $D(t)(0 \leq D(t) \leq 1)$. We assume that both modulation function $M(t)$ and demodulation function $D(t)$ are periodic functions with the same fundamental frequency $f_{0}$, i.e., the same period $T_{0}=1 / f_{0}$ (homodyne detection). The ideal (noise-free) measured intensity $B$ is given as

$$
B=\int_{T} E(t ; \tau) D(t) d t
$$

where $T \gg T_{0}$ is the sensor integration time. Next, we define $\mho(\tau)=\int_{T}(M \otimes h)(t ; \tau) D(t) d t$ as the fluorescence correlation function. It is described completely by the modulation and demodulation functions $M(t)$ and $D(t)$, respectively, and is a function only of the fluorescence lifetime $\tau$. Substituting Equation (1) and Equation (2) into Equation (3), the measured image intensity is given as

$$
B(\alpha ; \beta ; \tau)=\alpha \mho(\tau)+\beta,
$$

where $\beta=\gamma \int_{T} D(t) d t$ is the component of the measured intensity due to ambient light sources (other than the laser source). Equation (4) is the image formation equation of FD-FLIM. It states that the measured intensity is a function of three arguments: $\alpha$, $\beta$, and $\tau$. In general, all three are unknown. Therefore, $K(K \geq$ 3 ) intensity measurements are needed to recover the unknowns, where each measurement $B_{i}(1 \leq i \leq K)$ is taken by using a different pair of modulation and demodulation functions $M_{i}(t)$ and



(a) FD-FLIM

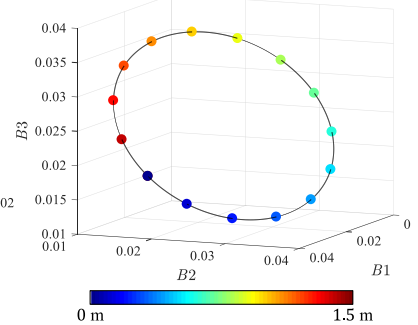

(b) C-ToF imaging
Fig. 3. The space of captured intensities for FD-FLIM and C-ToF imaging. (a) In FD-FLIM, the set of intensity points when the lifetime is varied forms an open 1D curve, with a non-uniform inter-point distance. (b) In C-ToF imaging, the set of intensity points when the scene depth is varied form a closed loop with a uniform inter-point distance.

$D_{i}(t)$, respectively. For example, one popular FD-FLIM measurement method is sinusoid coding, where both modulation and demodulation functions are sinusoids of the same frequency (Philip and Carlsson 2003). Different intensity measurements are taken by phase-shifting the demodulation function, while the modulation function remains the same.

Let the set of measured intensities be represented as a vector $\mathbf{B}=\left[B_{1}, B_{2}, \ldots, B_{K}\right]$ in the $K$-dimensional intensity space. For a given tuple of unknowns $\alpha, \beta$, and $\tau$, B is completely determined by the coding scheme $C$, which is defined as the set of modulation and demodulation functions $C=\left\{M_{i}(t), D_{i}(t)\right\}, 1 \leq i \leq K$. The analysis in the article is generally valid for the entire space of modulation and demodulation functions that are physically realizable (i.e., bounded amplitudes of modulation and demodulation functions), given a fixed light source power budget. Please refer to Section 6 for amplitude constraints for $C$. Further practical constraints can be imposed on $C$ due to the source peak power or system bandwidth. Figure 3(a) shows the set of intensity vectors B when $K=3$ and sinusoid is used for both modulation and demodulation functions. The locus of $\mathbf{B}$ is obtained by changing $\tau$ while fixing $\alpha$ and $\beta$.

Relationship to Phasor Plot Representation: The semi-circle shape in Figure 3(a) looks similar to the phasor plot often used to illustrate fluorescence emission with sinusoid illumination (Digman et al. 2008). However, while a phasor plot is the representation of amplitude attenuation and phase shift of sinusoid fluorescence emission, Figure 3(a) is the set of raw intensity values after integration of fluorescence emission with demodulation functions. In general, for a non-sinusoid coding scheme, the shape of the set of $\mathbf{B}$ is not a semi-circle.

Nomenclature. In the rest of the article, we will use the names of modulation and demodulation functions to denote a coding scheme. For example, "Sinusoid-Square" denotes a coding scheme that uses sinusoid modulation functions, and square demodulation functions.

\subsection{FD-FLIM vs. C-ToF Imaging}

Conceptually, the image formation model of FD-FLIM shares many aspects with that of continuous wave ToF (C-ToF) imaging (Bhandari et al. 2015a). Both imaging modalities require temporal coding of light intensities, and sinusoid coding is a well-established 
Table 1. Comparisons between FD-FLIM and C-ToF Imaging

\begin{tabular}{|c|c|c|}
\hline & C-ToF imaging & FD-FLIM \\
\hline Functional form of light incident on the sensor & Same as the light emitted by the source & Different functional form \\
\hline Space of intensity points when the lifetime or scene depth is varied & Closed loop with wrapping & Open curve \\
\hline Optimal modulation frequency & High frequencies & Function of the lifetime \\
\hline
\end{tabular}

coding scheme in both modalities. However, beyond these highlevel similarities, these two modalities have important differences, with different goals. The main difference in the image formation model is the impulse response $h(t)$ of the scene. As stated in Equation (1), the impulse response for FD-FLIM is an exponentially decaying function. In contrast, the impulse response for C-ToF is a shifted delta function $h(t)=\delta\left(t-\frac{2 d}{c}\right)$, where $d$ is the scene depth, and $c$ is the speed of light. This key difference in $h(t)$ leads to several distinctions between FD-FLIM and C-ToF imaging, as discussed below. The main differences are also summarized in Table 1 .

Functional Form of Reflected vs. Emitted Light. In C-ToF imaging, the light emitted from the source is time-shifted during travel from source to the scene and back, and thus, retains its functional form (e.g., a square wave remains a square wave). In contrast, in FD-FLIM, due to the exponential response function, the functional form of the fluorescence emission can be different from that of the incident light. As a result, the fluorescence lifetime information is encoded in not just the shift, but other properties of the waveform received at the sensor. For example, with sinusoid modulation functions, fluorescence lifetime information is encoded in both the amplitude and the phase shift of the emission signal. In contrast, in C-ToF, depth information is encoded in only the phase shift of the reflected signal. Please see the supplementary technical report for a detailed explanation.

The Space of Intensity Points When Lifetime or Scene Depth is Varied. Consider the locus of intensity points B for FD-FLIM, as $\tau$ is varied. The set of points form a 1D curve. Similarly, the set of intensities captured by a C-ToF imaging system, as scene depth is varied, also form a $1 \mathrm{D}$ curve in the intensity space (Gupta et al. 2018). For example, Figure 3(a) and (b) show example sets of intensity points B for FD-FLIM and C-ToF imaging (for $K=3$ sinusoidsinusoid coding), as $\tau$ and $d$ are varied, respectively. The key difference is that in FD-FLIM, the locus of $\mathbf{B}$ forms an open curve, but forms a closed loop in C-ToF imaging, which wraps around itself as $d$ is increased further. Therefore, while C-ToF based depth measurements are limited to a specific unambiguous depth range due to phase wrapping (Hansard et al. 2012), an FD-FLIM system, in principle, can recover the entire range of lifetimes $(\tau \in[0, \infty))$, without ambiguities, with as few as three measurements.

The Optimal Modulation Frequency. For C-ToF imaging, the distance between intensity points on the curve remains the same if $d$ increases by a constant amount, as shown in Figure 3(b). Also, the spacing between points increases uniformly over the entire curve, as the frequency of the modulation functions increases (Gupta et al. 2018; Lange 2000). On the other hand, in FD-FLIM, the distance between intensity points is non-uniform (exponentially decreasing as $\tau$ increases), as shown in Figure 3(a). Furthermore, as

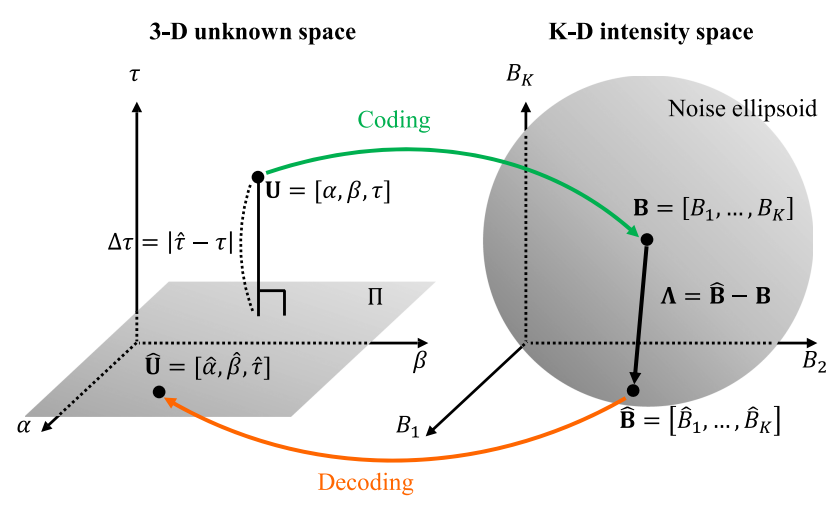

Fig. 4. Coding space theory of FD-FLIM. The 3D unknown space is the set of all possible unknown vectors $\{\mathrm{U}=[\alpha, \beta, \tau]\}$, and the $K$-D intensity space $(K \geq 3)$ is the set of corresponding intensity vectors $\{\mathbf{B}=$ $\left.\left[B_{1}, B_{2}, \ldots, B_{K}\right]\right\}$. A coding scheme maps $\mathbf{U}$ to $\mathbf{B}$, and the noise $\Lambda$ in $\mathbf{B}$ causes the lifetime error $\Delta \tau=|\hat{\tau}-\tau|$.

the modulation frequencies increase, the inter-point distance increases for relatively short $\tau$ 's, whereas it becomes shorter for relatively long $\tau$ 's. Therefore, the optimal frequency (in terms of lifetime recovery precision) in FD-FLIM is actually a function of the lifetime $\tau$. In contrast, in C-ToF imaging, higher modulation frequencies always result in higher depth precision, irrespective of the scene depths. Please see the supplementary technical report for details.

\section{MEAN LIFETIME ERROR}

In this section, we define a new measure to evaluate the performance of a given FD-FLIM coding scheme, based on the mean precision of the recovered lifetime. Consider the $3 \mathrm{D}$ space consisting of all possible unknown vectors $\{\mathrm{U}=[\alpha, \beta, \tau]\}$, as shown in Figure 4. A given coding scheme maps an unknown vector $\mathbf{U}$ in the unknown space to a true (without noise) intensity vector $\mathbf{B}=\left[B_{1}, B_{2}, \ldots, B_{K}\right](K \geq 3)$ in a $K$-dimensional intensity space. However, due to various sources of measurement noise (e.g., photon noise, sensor read noise, quantization noise), the sensor measures a noisy intensity vector $\hat{\mathbf{B}}=\mathbf{B}+\Lambda$ in the intensity space, where $\Lambda$ is the noise vector. A decoding algorithm then maps $\hat{\mathbf{B}}$ to an estimated unknown vector $\hat{\mathbf{U}}$, as shown in Figure 4 . Consider a plane $\Pi$ in the unknown space, parallel to the $\alpha$ and $\beta$ axes, and passing through the estimated unknown vector $\hat{U}$. Note that all the vectors on plane $\Pi$ correspond to the same lifetime $\tau$. Therefore, the shortest (perpendicular) distance from the true unknown point $\mathbf{U}$ to $\Pi$ is the lifetime estimation error $\Delta \tau=|\tau-\hat{\tau}|$, as shown in Figure 4.

Since noise vector $\Lambda$, and hence, the measured intensity vector $\hat{\mathbf{B}}$ are random variables, the expected lifetime error for a given 
unknown $\mathrm{U}=[\alpha, \beta, \tau]$ and coding scheme $C$ is given as

$$
\mathbb{E}[\Delta \tau](C)=\int_{\hat{\mathbf{B}}}|\tau-\hat{\tau}| p(\hat{\mathbf{B}} \mid \mathbf{B}(C)) d \hat{\mathbf{B}},
$$

where $p(\hat{\mathbf{B}} \mid \mathbf{B})$ is the probability density function of the measured noisy intensity $\hat{\mathbf{B}}$, given the true intensity $\mathbf{B}$, which is determined by $\mathbf{U}$ and $C . p(\hat{\mathbf{B}} \mid \mathbf{B})$ follows the $K$-dimensional normal distribution $^{3} \mathcal{N}(\mathrm{B}, \Sigma)$, where $\Sigma$ is a noise covariance matrix determined by the noise model, including photon noise and read noise (Hasinoff et al. 2010). For the sake of simplicity, we assume $\Sigma$ is diagonal. Equation (5) provides the expected lifetime error for a specific lifetime, corresponding to the unknown point $\mathbf{U}$. The mean lifetime error for a given range of lifetimes $\left[\tau_{1}, \tau_{2}\right]$ is given as

$\mathbb{E}_{\text {mean }}[\Delta \tau]\left(C ;\left[\tau_{1}, \tau_{2}\right]\right)=\frac{1}{\tau_{2}-\tau_{1}} \int_{\tau_{1}}^{\tau_{2}} \int_{\hat{\mathbf{B}}}|\tau-\hat{\tau}| p(\hat{\mathbf{B}} \mid \mathbf{B}(C)) d \hat{\mathbf{B}} d \tau$

Equation (5) is a special case of Equation (6) when $\tau_{1}=\tau_{2}=\tau$. The mean lifetime error is a measure of the performance of any given coding scheme, in terms of the precision of the recovered lifetime.

The mean lifetime error is a universal objective: given any coding scheme, the mean lifetime error can be numerically computed using the expression in Equation (6). Figure 6(a) shows the inverse mean lifetime error $\mathbb{E}_{\text {mean }}[\Delta \tau]^{-1}$ over the frequency $f_{0}$ for several coding schemes. Refer to Table 3 for the functional forms of each coding scheme. Based on the mean lifetime error, we define the optimal coding scheme $C_{\text {opt }}$ as the coding scheme that minimizes the mean lifetime error, over a specified range of lifetimes $\left[\tau_{1}, \tau_{2}\right]:$

$$
C_{o p t}=\arg \min _{C} \mathbb{E}_{m e a n}[\Delta \tau]\left(C ;\left[\tau_{1}, \tau_{2}\right]\right),
$$

where $\mathbb{E}_{\text {mean }}[\Delta \tau]\left(C ;\left[\tau_{1}, \tau_{2}\right]\right)$ is the mean lifetime error achieved by a coding scheme $C$ over the lifetime range $\left[\tau_{1}, \tau_{2}\right]$.

Computational Considerations for Mean Lifetime Error. The mean lifetime error derived in Equation (6) is generally applicable to the entire space of coding schemes, and thus can be used as a valuable analysis tool for evaluating the performance of different schemes. However, unfortunately, mean lifetime error does not have a closed-form analytical expression, and requires computationally intensive numerical computations, which involve the inverse problem of computing lifetime estimate $\hat{\tau}$ from the measured intensities.

In general, there is no closed-form solution for estimating $\hat{\tau}$, except for a few specific cases (e.g., Sinusoid - Sinusoid). Therefore, for most coding schemes, this decoding step requires an expensive iteration-based search procedure. Furthermore, the expression for the mean lifetime error requires high-dimensional numerical integration over the intensity space. As a result, estimating mean lifetime error, while conceptually simple, is prohibitively expensive from a computational standpoint for it to be used as an objective function in an efficient FD-FLIM coding optimization method.

\footnotetext{
${ }^{3}$ We assume that photon noise follows normal distribution. For a relatively large number of photon counts, Poisson distribution approaches the normal distribution, due to the central limit theorem.
}

\section{FAST SURROGATE FOR MEAN LIFETIME ERROR}

In this section, we derive a fast surrogate objective for the mean lifetime error, based on a first-order differential analysis of the FD-FLIM image formation model. The surrogate does not require computationally intensive decoding and integration, and thus, is significantly (three to four orders of magnitude) faster to compute as compared to the mean lifetime error, while accurately predicting the relative performance of different FD-FLIM coding schemes. Due to these properties, it can be used to efficiently explore and optimize over the space of coding schemes. In the following, we derive the surrogate.

First-Order Approximation of FD-FLIM Model. The mean lifetime error derived in the previous section (Equation (6)) is based on a relationship between intensity deviation (expressed as noise vector $\Lambda=\hat{\mathbf{B}}-\mathbf{B}$ ), and the resulting lifetime error $\Delta \tau=|\hat{\tau}-\tau|$. We can approximate the relationship between the intensity deviation and the lifetime error by taking the first-order partial derivative of the measured intensity $\mathbf{B}$ with respect to $\tau$ from the image formation model (Equation (4)). Let $\mathbf{B}_{\tau}=\partial \mathbf{B} / \partial \tau$ be the partial derivative of intensity vector $\mathbf{B}$ with respect to $\tau$. The $L_{2}$-norm of the vector $\mathbf{B}_{\tau}$ is given as $\left\|\mathbf{B}_{\tau}\right\|=\|\partial \mathbf{B}\| /|\partial \tau|$. Rearranging, we get a relationship between differential lifetime error $|\partial \tau|$ and the norm of the differential intensity variation vector $\partial \mathbf{B}=\left[\partial B_{1}, \ldots, \partial B_{K}\right]$ :

$$
|\partial \tau|=\frac{\|\partial \mathbf{B}\|}{\left\|\mathbf{B}_{\tau}\right\|} .
$$

Consider an intensity variation (noise) vector $\Lambda=\left[\Lambda_{1}, \ldots, \Lambda_{K}\right]$, where $\Lambda_{i}$ is the standard deviation of noise in $B_{i}(i=1, \ldots, K)$, the $i$ th intensity measurement. $\Lambda=\operatorname{diag}(\Sigma)$, where $\Sigma$ is the noise covariance matrix that can be computed from the intensity measurements B using the affine noise model. $\Lambda$ can be considered a representative (root-mean-square) intensity variation vector for the measurements. Then, by using the first-order approximation in the above equation, the first-order lifetime error $\Delta \tau_{f}$ due to intensity variation vector $\Lambda$ can be approximated as

$$
\Delta \tau_{f}=\frac{\|\Lambda\|}{\left\|\mathbf{B}_{\tau}\right\|} .
$$

With a slight abuse of notation, we define the mean $\Delta \tau_{f}$, over a range of lifetimes $\left[\tau_{1}, \tau_{2}\right]$ as

$$
\Delta \tau_{f}=\frac{1}{\tau_{1}-\tau_{2}} \int_{\tau_{1}}^{\tau_{2}} \frac{\|\Lambda\|}{\left\|\mathbf{B}_{\tau}\right\|} d \tau
$$

The first-order lifetime error $\Delta \tau_{f}$ can be computed from vectors $\Lambda$ and $\mathbf{B}_{\tau}$, both of which can be estimated with minimal computations directly from the image formation equation, without computationally expensive inverse decoding or numerical integration required to estimate the mean lifetime error. ${ }^{4}$ However, unfortunately, $\Delta \tau_{f}$ does not accurately predict the coding scheme performance. Figure 6(a) and (b) compare the mean lifetime error $\mathbb{E}_{\text {mean }}[\Delta \tau]$ and the first-order lifetime error $\Delta \tau_{f}$, as a function of modulation frequency, for several coding schemes. All the values

\footnotetext{
${ }^{4} \mathbf{B}_{\tau}$ can be estimated analytically as $\mathbf{B}_{\tau}=\alpha \mho^{\prime}(\tau)$ from Equation (4), if $\mho^{\prime}(\tau)$, the gradient of the fluorescence correlation function, can be expressed in an analytical form. In general, $\mathbf{B}_{\tau}$ can be computed numerically by finite element approximation as $(\mathbf{B}(\tau+\Delta \tau)-\mathbf{B}(\tau)) / \Delta \tau$, with an infinitesimal $\Delta \tau$. In our computations, we used the numerical approach with $\Delta \tau=0.001 \mathrm{~ns}$.
} 


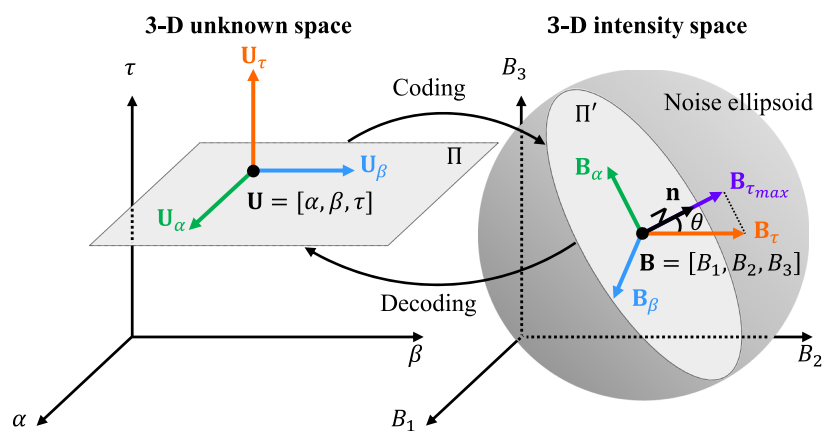

Fig. 5. Surrogate derivation. The direction $\mathbf{n}$ along which the variation in $\mathbf{B}$ leads to the maximum variation in decoded lifetime. The maximum differential lifetime error can be approximated by the norm of the noise variance along $\mathbf{n}$ divided by the norm of $\mathbf{B}_{\tau_{\max }}$, which is the projection of $\mathbf{B}_{\tau}=\partial \mathbf{B} / \partial \tau$ onto $\mathbf{n}$. The surrogate is the average of the maximum differential lifetime error over a range of lifetimes.

are computed at a given lifetime range. For ease of visualization, the inverse values $\mathbb{E}_{\text {mean }}[\Delta \tau]^{-1}$ and $\Delta \tau_{f}^{-1}$ are plotted. As can be noticed, there is no strong correlation between the values of the two objectives. Thus, while $\Delta \tau_{f}$ is fast to compute, it cannot be used as a surrogate objective function for coding scheme optimization.

Geometrical Analysis of FD-FLIM Imaging Model. The main reason behind weak correlation between $\mathbb{E}_{\text {mean }}[\Delta \tau]$ and $\Delta \tau_{f}$ is the following. The approximation in Equation (9) implicitly assumes that the noise vector $\Lambda$ and the intensity derivative vector $\mathbf{B}_{\tau}$ point in the same direction. In general, this is not true. Therefore, we need to find a representative direction in the intensity space, variation along which can be accurately related to the lifetime error.

Consider vectors $\mathbf{B}_{\alpha}$ and $\mathbf{B}_{\beta}$, defined as the partial derivatives of the intensity vector $\mathbf{B}$ with respect to the unknowns $\alpha$ and $\beta$, respectively:

$$
\mathbf{B}_{\alpha}=\frac{\partial \mathbf{B}}{\partial \alpha}=\mho(\tau)
$$

and

$$
\mathbf{B}_{\beta}=\frac{\partial \mathbf{B}}{\partial \beta}=\mathbf{1},
$$

where $\mathbf{1}$ is a vector whose elements are all 1's. Let $\Pi^{\prime}$ be the plane defined by vectors $\mathbf{B}_{\alpha}$ and $\mathbf{B}_{\beta}$ in the intensity space. The key observation is that any intensity variation (due to noise) within $\Pi^{\prime}$ results in zero lifetime error. This is because intensity changes along $\mathbf{B}_{\alpha}$ (or $\mathbf{B}_{\beta}$ ) corresponds to change in $\alpha$ (or $\beta$ ) in the unknown space, but $\tau$ remains constant. Let $\mathbf{n}$ be the unit vector perpendicular to plane $\Pi^{\prime}$, as shown in Figure 5:

$$
\mathbf{n}=\frac{\mathbf{B}_{\alpha} \times \mathbf{B}_{\beta}}{\left\|\mathbf{B}_{\alpha} \times \mathbf{B}_{\beta}\right\|},
$$

where $\times$ is the cross product. Since the variation in $\mathbf{B}$ along $\mathbf{n}$ leads to the maximum variation in decoded lifetime $\hat{\tau}$ (thus, maximum lifetime error), we compute the differential lifetime error $|\partial \tau|$ from $\|\partial \mathbf{B}\|$ along $\mathbf{n}$. To this end, we define $\mathbf{B}_{\tau_{\max }}$ as the projection of $\mathbf{B}_{\tau}=\partial \mathbf{B} / \partial \tau$ onto $\mathbf{n}:$

$$
\mathbf{B}_{\tau_{\max }}=\left\|\mathbf{B}_{\tau}\right\| \mathbf{n} \cos \theta,
$$

where $\theta$ is the angle between $\mathbf{n}$ and $\mathbf{B}_{\tau}$. Similarly, we define the noise variance along $\mathbf{n}$ as

$$
\Lambda_{\mathbf{n}}=\Lambda \circ \mathbf{n}
$$

where $\circ$ is the element-wise vector multiplication operator. Then, substituting $\mathbf{B}_{\tau_{\max }}$ and $\Lambda_{\mathbf{n}}$ for $\mathbf{B}_{\tau}$ and $\Lambda$, respectively, in Equation (10), we get

$$
\Delta \tau_{S}=\frac{1}{\tau_{2}-\tau_{1}} \int_{\tau_{1}}^{\tau_{2}} \frac{\left\|\Lambda_{\mathbf{n}}\right\|}{\left\|\mathbf{B}_{\tau_{\max }}\right\|} d \tau .
$$

This is an important equation; it provides the expression for the proposed surrogate of mean lifetime error. Figure 6(c) shows the inverse surrogate values $\Delta \tau_{S}^{-1}$ for the same coding schemes and lifetime ranges as Figure 6(a). As can be noticed, there is a strong correlation between the values of the mean lifetime error and surrogate, for all the coding schemes, over the entire range of modulation frequencies. Figure 7 shows that the surrogate can predict the coding scheme performance well even with much larger lifetime ranges. For two lifetime ranges, $\left[\tau_{1}, \tau_{2}\right]=[0.5 \mathrm{~ns}, 10 \mathrm{~ns}]$ and $\left[\tau_{1}, \tau_{2}\right]=[5 \mathrm{~ns}, 50 \mathrm{~ns}]$, the surrogate shows high correlation with mean lifetime error. Note that the surrogate approximates the mean lifetime error only up to a scale factor. Although the surrogate cannot be used to directly predict the mean lifetime error, it can be used to compare relative performance of coding schemes since the scale factor is the same for different coding schemes. Consequently, we use this surrogate as an objective function to find the optimal coding scheme, instead of the computationally intensive mean lifetime error. For more comparison results between the surrogate and the mean lifetime error with other lifetime ranges, please see the supplementary technical report.

Runtime Comparison. The surrogate (Equation (16)) can be computed via a small number of analytic lightweight computations. Specifically, $\mathbf{n}$ can be computed from Equations (11) and (12). The noise vector $\Lambda$ and gradient $\mathbf{B}_{\tau}$ can be computed from $\mathbf{B}$, which in turn can be used to compute the projections $\Lambda_{\mathbf{n}}$ and $\mathbf{B}_{\tau_{\text {max }}}$, as given in Equations (14) and (15). There is no computationally expensive high-dimensional integration and lifetime decoding procedures, as required for the mean lifetime error computation. Table 2 shows the runtime comparison between the mean lifetime error and the surrogate for the coding schemes used in Figure 6 when the modulation frequency $f_{0}=1.6 \mathrm{MHz}$. Computation of the surrogate is three orders of magnitude faster than the mean lifetime error, while maintaining high correlation with the mean lifetime error, as shown in Figure 6.

\section{CODING SCHEME OPTIMIZATION}

In this section, we demonstrate the capability of the surrogate for FD-FLIM coding scheme optimization. The surrogate is general in its scope, and could be used as an objective function in a broad range of derivative-free optimization approaches, including based on pattern search (Torczon 1997), genetic algorithms (Deb et al. 2002), and Nelder-Mead method (Nelder and Mead 1965). For ease of implementation, we adopt a simple search-based optimization approach as a proof-of-concept.

Search-Based Optimization. In principle, the space of coding functions is infinite dimensional. To keep the optimization 


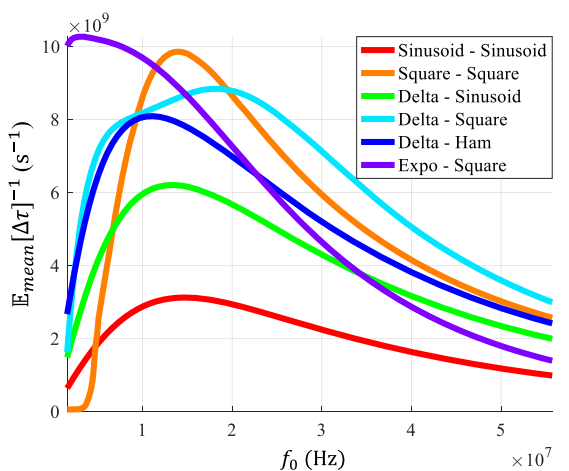

(a) Inverse mean lifetime error

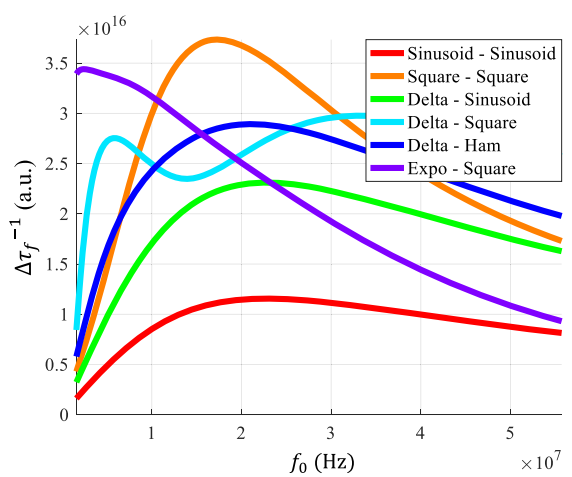

(b) Inverse $\Delta \tau_{f}$

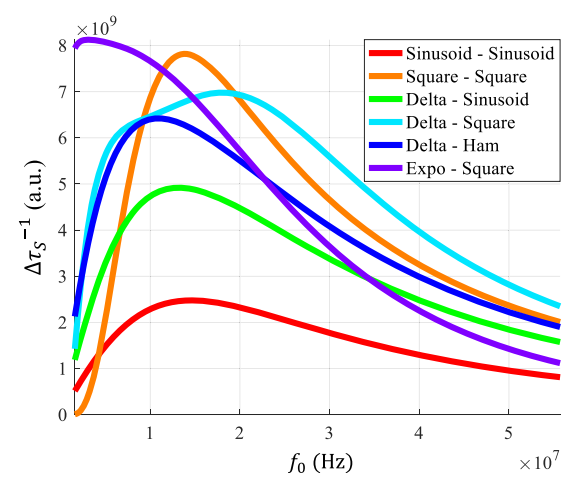

(c) Inverse surrogate value

Fig. 6. Coding scheme performance comparison according to different objectives. (a) Inverse mean lifetime errors $\mathbb{E}_{\text {mean }}[\Delta \tau]^{-1}$, (b) inverse values of the first-order lifetime error $\Delta \tau_{f}^{-1}$, and (c) inverse surrogate values $\Delta \tau_{S}^{-1}$. The performance of several coding schemes is compared as a function of the fundamental modulation frequency $f_{0}$, for a given lifetime range $\left[\tau_{1}, \tau_{2}\right]=[6 \mathrm{~ns}, 8 \mathrm{~ns}]$ and $K=3$. The surrogate values have a strong correlation with the mean lifetime errors across various coding schemes and frequencies, and thus, can be used as a lightweight alternative to the computationally intensive mean lifetime error, in order to find the optimal coding scheme efficiently.

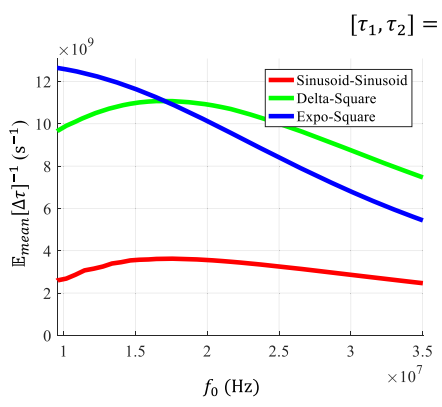

(a) Inverse mean lifetime error

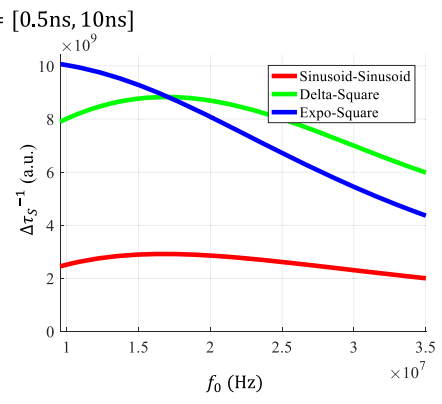

(b) Inverse surrogate value

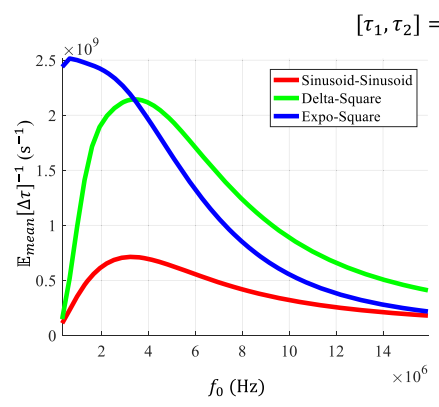

(c) Inverse mean lifetime error

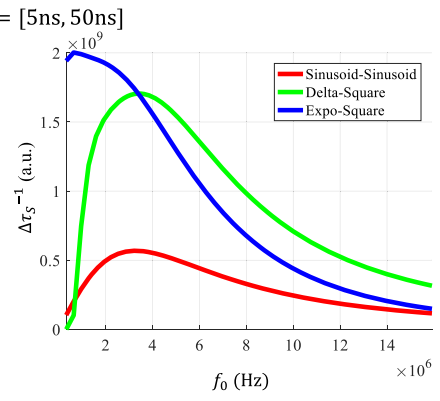

(d) Inverse surrogate value

Fig. 7. Coding scheme performance comparison with relatively large lifetime ranges. Sinusoid-Sinusoid, Delta-Square, and Expo-Square are compared in terms of (a) inverse mean lifetime errors $\mathbb{E}_{\text {mean }}[\Delta \tau]^{-1}$, and (b) inverse surrogate values $\Delta \tau_{S}^{-1}$ when the lifetime range is $\left[\tau_{1}, \tau_{2}\right]=[0.5 \mathrm{~ns}, 10 \mathrm{~ns}]$, and also in terms of (c) inverse mean lifetime errors $\mathbb{E}_{\text {mean }}[\Delta \tau]^{-1}$, and (d) inverse surrogate values $\Delta \tau_{S}^{-1}$ when the lifetime range is $\left[\tau_{1}, \tau_{2}\right]=[5 \mathrm{~ns}, 50 \mathrm{~ns}]$. The surrogate accurately predicts the coding scheme performance even for relatively large lifetime ranges.

Table 2. Runtime Comparison between the Mean Lifetime Error and the Surrogate

\begin{tabular}{ccc}
\hline Schemes & \multicolumn{2}{c}{ Runtime $(s)$} \\
& Mean lifetime error & Surrogate \\
\hline Sinusoid-Sinusoid & $3.58 \times 10^{1}$ & $2.60 \times 10^{-2}$ \\
Square-Square & $0.52 \times 10^{1}$ & $0.87 \times 10^{-2}$ \\
Delta-Sinusoid & $3.08 \times 10^{1}$ & $0.73 \times 10^{-2}$ \\
Delta-Square & $2.58 \times 10^{1}$ & $0.54 \times 10^{-2}$ \\
Delta-Ham & $1.31 \times 10^{1}$ & $1.21 \times 10^{-2}$ \\
Expo-Square & $1.57 \times 10^{1}$ & $0.62 \times 10^{-2}$ \\
\hline
\end{tabular}

The surrogate is up to three orders of magnitude faster to compute as compared to the mean lifetime error.

tractable, we narrow the space to a finite set of functions. We consider coding schemes created using pairs of modulation and demodulation functions derived from a fixed library of functions, including sinusoid, square waves, impulse train, exponential functions (Aljunid et al. 2013), and trapezoidal Hamiltonian function (Gupta et al. 2018).
Table 3. Functions Used for Coding Schemes in Optimization

\begin{tabular}{lc}
\hline Functions & Equations \\
\hline Sinusoid & $E_{0}\left(1+\cos \left(2 \pi f_{0} t\right)\right)$ \\
Square & $E_{0}\left(1+\operatorname{sqr}\left(2 \pi f_{0} t, \chi\right)\right)$ \\
Delta & $E_{0} \sum_{n=-\infty}^{\infty} \delta\left(t-n T_{0}\right)$ \\
Expo & $E_{0} e^{\kappa t}, \kappa>0,0 \leq t \leq T_{0}$ \\
Ham & Hamiltonian functions (Gupta et al. 2018) \\
\hline
\end{tabular}

The modulation frequency $f_{0}$, duty cycle $\chi$ of the Square function, and the exponent $\kappa$ of the Expo function are the function parameters that are optimized. The scale factor $E_{0}$ is chosen appropriately to satisfy the constraints (constant average source power, non-negativity) on the modulation and the demodulation functions.

Table 3 provides a list of the functional forms and the corresponding mathematical expressions. sqr is the square function whose amplitude is between -1 and $1 . \chi$ is the duty cycle of sqr function. The amplitudes $E_{0}$ of all functions are scaled appropriately such that the average source power is the same for all the modulation functions, and the amplitudes of the demodulation functions are between 0 and 1 . Please refer to 
the supplementary technical report for details. Specifically, we consider 12 pairs of modulation and demodulation functions: For modulation functions $M(t)$, we use sinusoidal, square, delta, and exponentially rising functions. For demodulation functions $D(t)$, we use sinusoidal, square, and trapezoidal Hamiltonian functions. We use the notation Sinusoid, Square, Delta, Expo, and Ham for sinusoidal, square, delta, exponentially rising, and Hamiltonian functions, respectively.

Importance of Optimizing Functional Parameters. Each functional form (e.g., sinusoid, square, exponential) is described by a set of parameters, such as modulation frequency $f_{0}$ (period $T_{0}$ ), duty cycle $\chi$ for the square waves, and exponent $\kappa$ for the exponential function. The performance (mean lifetime error) of a coding scheme has a strong dependence on the functional parameters. For example, Figure 6(a) shows the mean lifetime error of different schemes varies significantly as the modulation frequency is varied. Furthermore, the optimal modulation frequency (location of the peak of the curves) is different for different coding schemes, and it also depends on the range of lifetimes $\left(\left[\tau_{1}, \tau_{2}\right]\right)$ used to compute the errors. Therefore, given an application with a given range of lifetimes to be measured, in order to determine the optimal scheme, it is critical to compute the optimal functional parameters.

Despite limiting the coding schemes to a finite set of functional forms, due to the parameter optimization, the search space is highdimensional. As a result, developing an efficient optimization procedure that can estimate globally optimal solution remains challenging. One solution is to perform an exhaustive search in the parameter space. Such an exhaustive search, while conceptually simple, is prohibitively expensive from a computational standpoint if the mean lifetime error is used as the objective function. However, since the proposed surrogate is computationally lightweight, we show that it is possible to perform an exhaustive search-based optimization using the surrogate as an objective function, and determine the globally optimal solution. It may be possible to use the surrogate to design more efficient optimization procedures. Developing such optimization algorithms based on the proposed surrogate may enable optimization over a larger search space (e.g., more functional forms), and is a promising future research direction.

Our search-based algorithm has two steps. First, the optimal parameter set $\mathcal{P}_{o p t}$ for each coding scheme is obtained by minimizing the surrogate:

$$
\mathcal{P}_{o p t}=\arg \min _{\mathcal{P}} \Delta \tau_{\mathcal{S}}(\mathcal{P})
$$

For example, the optimal parameters for the Expo-Square coding scheme are determined by performing a search over the 3D parameter space $\mathcal{P}=\left\{f_{0}, \kappa, \chi\right\}$; the three parameters are the fundamental modulation frequency $f_{0}$, the exponent $\kappa$ of the exponential function, and duty cycle $\chi$ of the square wave. Figure 8(a) shows $\Delta \tau_{\mathcal{S}}^{-1}$ over the 2D parameter space of the Expo-Square coding scheme. The lifetime range is [4ns, 6ns]. Although the search is performed over a 3D space, we show the search results over a $2 \mathrm{D}$ space for visualization. As seen from the figure, the surrogate varies significantly over the parameter space, and is a non-convex function of the parameters. Since the surrogate is fast to compute, we can compute the optimal solution via an exhaustive search.

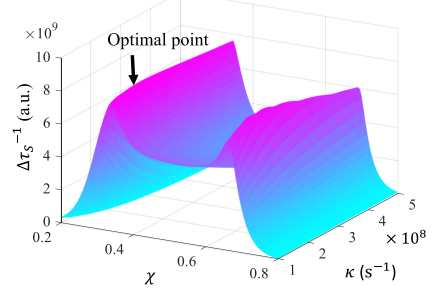

(a) Optimization over Expo - Square

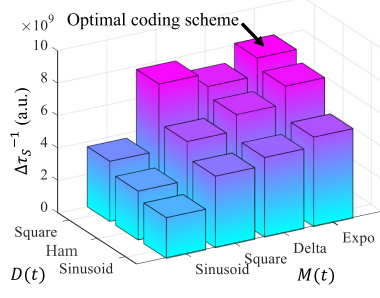

(b) Optimization over various coding schemes
Fig. 8. Optimization. (a) Inverse surrogate values over two parameters of Expo-Square, the exponent $\kappa$, and the duty cycle $\chi$. The optimal parameters can be found by minimizing the surrogate (or maximizing the inverse surrogate). (b) The optimum inverse surrogate values for various coding schemes. The optimum value for each coding scheme is determined by finding its optimal parameters.

In the second step, the optimal coding scheme $C_{o p t}$ is determined by simply finding the scheme (with the optimized parameters) that achieves the minimum value of the surrogate:

$$
C_{o p t}=\arg \min _{C} \Delta \tau_{\mathcal{S}}\left(C\left(\mathcal{P}_{o p t}\right)\right) .
$$

Figure 8 (b) shows $\Delta \tau_{\mathcal{S}}^{-1}$ over the search space where each coding scheme's parameters are optimized. Among the 12 coding schemes considered in our search space, the Expo-Square scheme achieves the best performance, with three times higher precision as compared to the conventional Sinusoid-Sinusoid coding scheme. ExpoSquare denotes the coding scheme with exponential modulation, and square demodulation function. The explicit forms of these functions are listed in Table 3. The relevant parameters of ExpoSquare are $\kappa$, the exponent of the modulation function, $\chi$, the duty cycle of the demodulation function, and $f_{0}$, the modulation frequency. For detailed equations, please refer to the supplementary technical report.

Dependence of Relative Coding Scheme Performance on Lifetime Range. The relative coding scheme performance depends on the range of lifetimes considered during the optimization. Given the low computational complexity of the proposed surrogate, it is possible to perform such optimization for any set of coding schemes with a different set of parameters, and for a different range of lifetimes, depending on the application. For more optimization results with other lifetime ranges, please see the supplementary technical report.

\subsection{Unipolar and Bipolar Demodulation Functions}

Demodulation functions in FD-FLIM can be unipolar $(0 \leq D(t) \leq$ $1)$ or bipolar $(-1 \leq D(t) \leq 1)$. For example, a FD-FLIM system using an image intensifier can implement unipolar (positive) demodulation functions, such as a positive sinusoid, $0.5+0.5 \cos \left(2 \pi f_{0} t\right)$. On the other hand, a lock-in amplifier-based FD-FLIM system can implement bipolar demodulation functions, such as a zeromean sinusoid, $\cos \left(2 \pi f_{0} t\right)$. Lock-in detection, in general, has better photon efficiency than image intensifiers (Philip and Carlsson 2003), since bipolar functions use the entire incident flux for FLIM estimation.

We compare the performance between unipolar and bipolar demodulation functions for the Sinusoid-Sinusoid and Expo-Square 


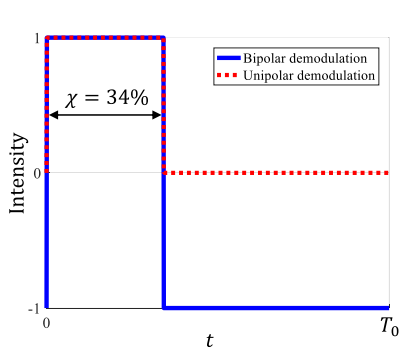

(a) Unipolar and bipolar demodulation functions for Expo-Square

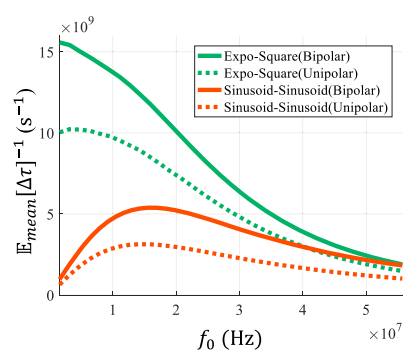

(b) Inverse mean lifetime error with two demodulation functions
Fig. 9. Performance comparison between unipolar and bipolar demodulation functions. Coding schemes with Unipolar and bipolar demodulation functions are compared in terms of mean lifetime error $\left(\mathbb{E}_{\text {mean }}[\Delta \tau]\right)$. Sinusoid-Sinusoid and Expo-Square coding schemes are used for comparison, for $\tau=7 \mathrm{~ns}$. The optimal parameters for Expo-Square with unipolar demodulation function is also used for the bipolar demodulation case for comparison. Figure 9(a) shows unipolar and bipolar demodulation functions used for Expo-Square. As shown in Figure 9(b), coding schemes with bipolar demodulation functions show better performance.

coding schemes. For Expo-Square with unipolar demodulation function, two parameters, $\kappa$ and $\chi$ are optimized by surrogate, and the same parameters are used for the bipolar demodulation case for comparison. Figure 9(a) shows unipolar and bipolar demodulation functions used for Expo-Square. As shown in Figure 9(b), coding schemes with bipolar demodulation functions achieve better performance than unipolar demodulation functions. This is due to efficient use of photons for both positive and negative lobes as well as increased amplitudes in the case of bipolar demodulation functions. It is interesting to note that Expo-Square with unipolar demodulation performs better as compared to Sinusoid-Sinusoid with bipolar demodulation, at their respective optimal frequencies. For the following analysis, we limit our scope to unipolar demodulation functions for ease of analysis and noise standard deviation computation. The same analysis can be extended to bipolar demodulation functions.

\subsection{Robustness of Coding Schemes to Signal Distortions}

In practice, it is challenging to create perfect desired signal shapes for coding schemes due to hardware limitations. The distortion in signal shapes for coding schemes degrades coding scheme performance. We tested robustness of coding schemes to signal shape distortion. We model the hardware impulse response causing signal distortion as a low-pass (Gaussian) filter. We distort the modulation and demodulation functions by applying Gaussian filters with different sizes. The size of the Gaussian filter (amount of low-pass filtering) is changed from $0 \%$ to $20 \%$, where $20 \%$ means that the filter size is $20 \%$ of a modulation period of Expo-Square. For each amount of low-pass filtering, the same size of the filter is applied to all coding schemes. The correlation values are obtained with these distorted coding schemes and photon noise is added to the measurements. Figure 10(a) shows distorted modulation and demodulation functions for Expo-Square, with three different amounts of low-pass filtering. For distorted waveforms, we used look-up tables (built by calibrating the system's distorted impulse response) for decoding lifetimes. Figure 10(b) shows the

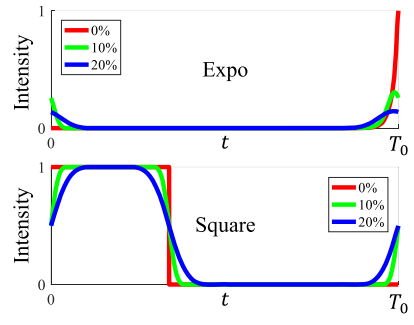

(a) Distorted Expo-Square coding scheme

Fig. 10. Robustness of coding schemes to signal distortion. Coding scheme performance is compared when different amounts of low-pass filtering is applied to either modulation or demodulation function. Photon noise is added to the measurements and a look-up table for decoding lifetime is created by calibration. Inverse mean lifetime error is compared between coding schemes. The performance of every scheme degrades as the distortion level increases.

inverse mean lifetime error when the amount of low-pass filtering changes from $0 \%$ to $20 \%$. The performance of each coding scheme degrades as the distortion level increases.

Different Types of Signal Distortion and Decoding Without Calibration. In some scenarios, the signal distortion is not modeled as a low-pass filtering. For example, often digital circuits are used for generating demodulation functions in a sinusoid coding scheme. The resulting demodulation function (ideally a square wave) has extra harmonics, resulting in a distorted sinusoid. However, the extra harmonics are canceled out by the sinusoid modulation function in correlation. In this case, the lifetime can be estimated by sinusoid coding scheme without calibration.

\section{VALIDATION BY SIMULATIONS}

In this section, we use simulations to demonstrate the capability of the proposed surrogate as an analysis and design tool for FDFLIM coding design. To this end, we evaluated the performance of various FD-FLIM coding schemes via simulations, and compared the results with our surrogate prediction results. As example test cases, we use the fluorescence lifetime data of the brain tissue (Figure 11(a) and (b)) which is used to characterize metabolic changes for Glioblastoma Multiforme (a type of brain cancer) study and another data of cells (Figure 11(c)) where fat cells are differentiated from other cells by lifetime. The ground-truth fluorescence lifetime image was acquired using a TD-FLIM approach. The size of the image is $256 \times 256$. Since the lifetime range for Figure 11(a) is relatively short, we artificially increased the lifetime range linearly in Figure 11(b) to evaluate the performance of coding schemes on samples with relatively large lifetime range. For each pixel and each coding scheme, we compute $K=3$ intensity values as captured by the sensor, based on the the image formation model (Equation (4)) with $\alpha=48.6, \gamma=5.1$, and $T=0.1 \mathrm{~s}$. For Figure 11(b), relatively short integration time, $T=0.005 \mathrm{~s}$, is used to highlight performance difference in longer lifetime range. The values of $\alpha$ and $\gamma$ depend on imaging system properties, and were computed based on typical FD-FLIM system parameters (Zhao et al. 2011). Finally, we add noise to the computed intensity values, 

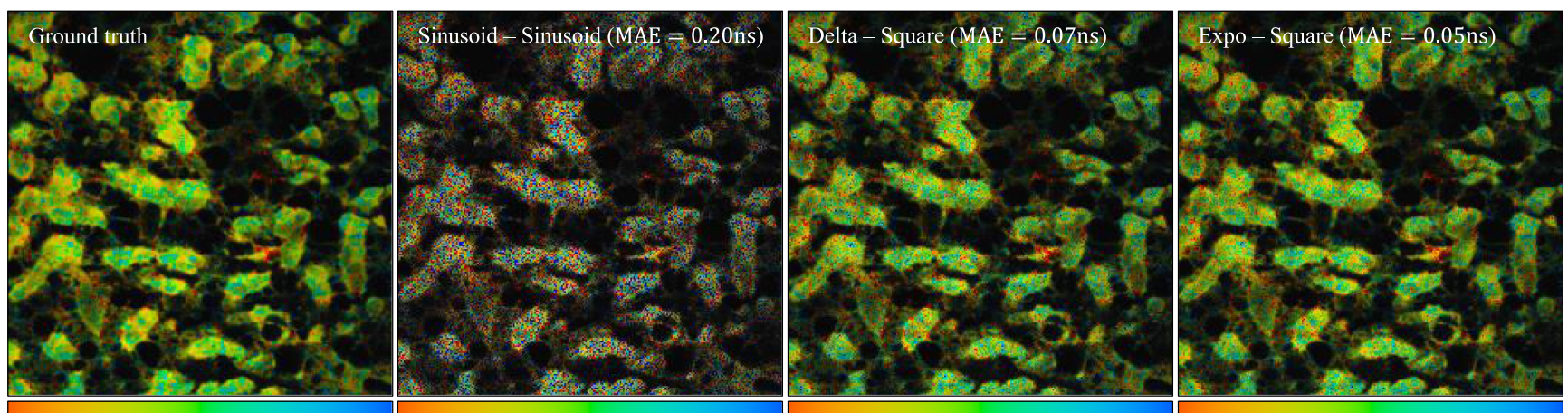

$1.70 \mathrm{~ns} 1.25 \mathrm{~ns}$

$1.70 \mathrm{~ns} 1.25 \mathrm{~ns}$

$1.70 \mathrm{~ns}$

(a) Brain tissue in original lifetime range
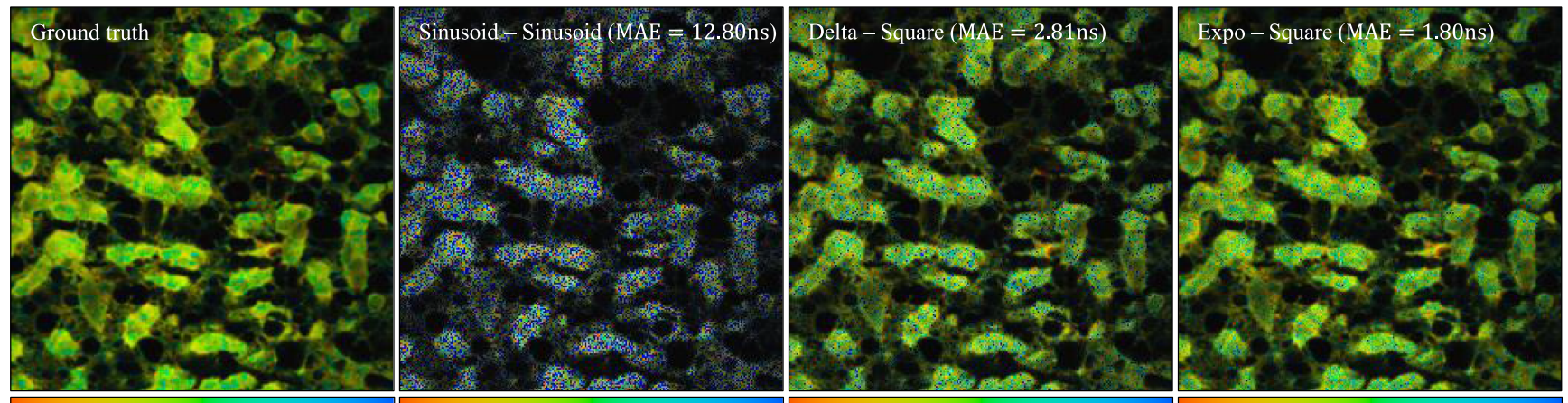

$1.50 \mathrm{~ns}$

$15.00 \mathrm{~ns} 1.50 \mathrm{~ns}$

$15.00 \mathrm{~ns} 1.50 \mathrm{~ns}$

$15.00 \mathrm{~ns} 1.50 \mathrm{~ns}$

$15.00 \mathrm{~ns}$

(b) Brain tissue in artificially increased lifetime range

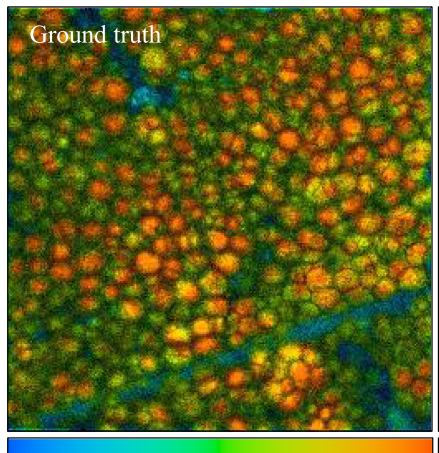

$1.00 \mathrm{~ns}$

$5.00 \mathrm{~ns} 1.00 \mathrm{~ns}$
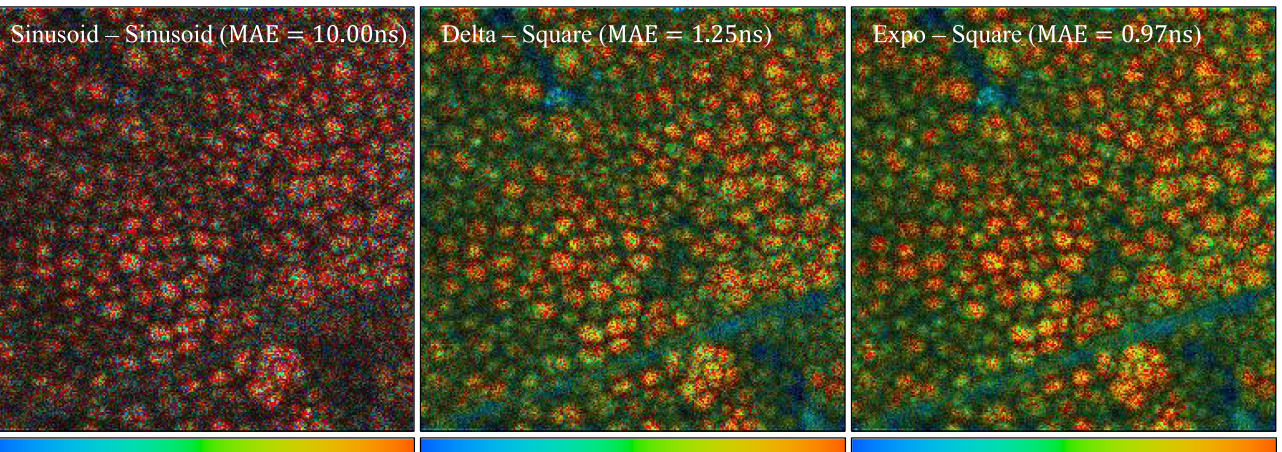

(c) Adipose tissue (body fat)

Fig. 11. Simulation-based comparisons between different coding schemes. Given ground truth fluorescence lifetime data, fluorescence lifetime images are reconstructed using Sinusoid-Sinusoid, Delta-Square, and Expo-Square from the simulated captured intensities of brain tissue in original lifetime range (a), same tissue in artificially increased lifetime range (b), and adipose tissue (c). In all cases, Expo-Square shows the best performance.

where the noise variance is estimated from the affine noise model, including photon noise and read noise (Hasinoff et al. 2010).

Decoding. In the decoding stage, lifetime is estimated for each pixel from the $K$ noisy intensity values, and shown as a colorcoded fluorescence lifetime image. For $K=3$ measurements, from Equation (4), we can eliminate the unknowns $\alpha$ and $\beta$ as follows:

$$
F(\tau)=\frac{\mho_{1}(\tau)-\mho_{2}(\tau)}{\mho_{2}(\tau)-\mho_{3}(\tau)}=\frac{B_{1}-B_{2}}{B_{2}-B_{3}},
$$

where $F(\tau)$ is a known function determined by a coding scheme. Given three intensity values $B_{1}, B_{2}$, and $B_{3}$, we can estimate $\tau$ by solving Equation (19). Refer to the supplementary technical report for details.

We compared three coding schemes, Sinusoid-Sinusoid, perhaps the most widely used FD-FLIM coding scheme (Philip and Carlsson 2003), Delta-Square, a high-performance FD-FLIM coding scheme proposed recently in literature (Schlachter et al. 2009), and Expo-Square, which is the optimal coding scheme according to our surrogate computation. The optimal parameters $\mathcal{P}_{\text {opt }}$ for these coding schemes were computed using the surrogate for the given lifetime ranges of the ground truth. The lifetime ranges are $[1.17 \mathrm{~ns}, 1.82 \mathrm{~ns}],[0.3 \mathrm{~ns}, 16 \mathrm{~ns}]$, and [1ns, 5ns] for Figure 11(a), 
Table 4. Estimated Optimal Parameter Sets

\begin{tabular}{lccc}
\hline & Sinusoid-Sinusoid & Delta-Square & Expo-Square \\
\hline Brain tissue of Figure 11(a) & $\left\{f_{0}=72.7 \mathrm{MHz}\right\}$ & $\left\{f_{0}=5.1 \mathrm{MHz}, \chi=0.34\right\}$ & $\left\{f_{0}=8.9 \mathrm{MHz}, \kappa=1.1 e 9 \mathrm{~s}^{-1}, \chi=0.34\right\}$ \\
Brain tissue of Figure 11(b) & $\left\{f_{0}=11.1 \mathrm{MHz}\right\}$ & $\left\{f_{0}=10.8 \mathrm{MHz}, \chi=0.36\right\}$ & $\left\{f_{0}=3.0 \mathrm{MHz}, \kappa=0.19 e 9 \mathrm{~s}^{-1}, \chi=0.35\right\}$ \\
\multicolumn{1}{c}{ Adipose tissue } & $\left\{f_{0}=32.9 \mathrm{MHz}\right\}$ & $\left\{f_{0}=27.2 \mathrm{MHz}, \chi=0.39\right\}$ & $\left\{f_{0}=4.1 \mathrm{MHz}, \kappa=0.52 e 9 \mathrm{~s}^{-1}, \chi=0.34\right\}$ \\
\hline
\end{tabular}

The optimal parameter sets of three coding schemes, for different tissues, were computed using our surrogate for simulations.

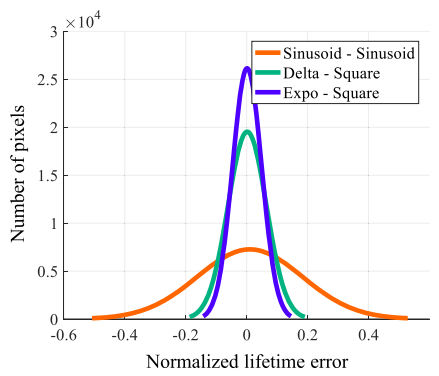

(a) Histograms of the normalized lifetime errors

\begin{tabular}{|c|c|c|}
\hline Coding schemes & $\overline{\mathrm{MAE}}$ & $\overline{\Delta \tau_{S}}$ \\
\hline Sinusoid - Sinusoid & 1.000 & 1.000 \\
\hline Delta - Square & 0.365 & 0.370 \\
\hline Expo - Square & 0.278 & 0.282 \\
\hline
\end{tabular}

(b) Standard deviations of the normalized lifetime errors and the surrogate values

Fig. 12. Statistical results of the simulation and comparison with the surrogate values. (a) Histograms of the normalized lifetime errors $((\tau-\hat{\tau}) / \tau)$ for different coding schemes. (b) Comparison of the normalized mean absolute errors, $\overline{\mathrm{MAE}}$ with the surrogate values $\overline{\Delta \tau_{\mathcal{S}}}$. The values are normalized such that both values for Sinusoid-Sinusoid are 1. There is a strong correlation between $\overline{\sigma_{\Delta \tau}}$ and $\overline{\Delta \tau_{\mathcal{S}}}$.

(b), and (c), respectively. The optimal parameters for the different schemes and different tissues are listed in Table 4. Note that the optimal parameters for different schemes and different tissues are significantly different.

Figure 11 shows the comparison between the fluorescence lifetime images obtained by three coding schemes. The black pixels in the images denote a region with no lifetime information. The result with the Sinusoid-Sinusoid scheme is noisy, with low SNR. In contrast, the lifetime image achieved by Delta-Square and Expo-Square is closer to ground truth, with lower noise. Figure 12(a) shows the histograms of normalized lifetime errors $(\tau-\hat{\tau}) / \tau$ of the three coding schemes for brain tissue in original lifetime range (Figure 11(a)). Expo-Square achieves the best performance in terms of estimated lifetime precision and SNR. Figure 12(b) shows the comparison between the mean absolute errors $\overline{\mathrm{MAE}}$ and the surrogate values $\overline{\Delta \tau_{\mathcal{S}}}$. Both $\overline{\mathrm{MAE}}$ and $\overline{\Delta \tau_{\mathcal{S}}}$ are normalized such that the corresponding values for the SinusoidSinusoid scheme is 1 . As shown in the table, $\overline{\mathrm{MAE}}$ and $\overline{\Delta \tau_{\mathcal{S}}}$ show strong correlation, thus suggesting that the proposed surrogate can be used as an easy-to-compute and accurate objective function for coding scheme optimization.

\section{HARDWARE PROTOTYPE AND EXPERIMENTS}

\subsection{Hardware Prototype}

We developed a proof-of-concept hardware prototype that can implement various FD-FLIM coding schemes (Figure 13). Since most off-the-shelf FD-FLIM systems use a fixed coding scheme, we develop a single-pixel-based prototype that can admit arbitrary waveforms. A lifetime image of a sample is created by moving the sample on translation stages, or by scanning light beam via

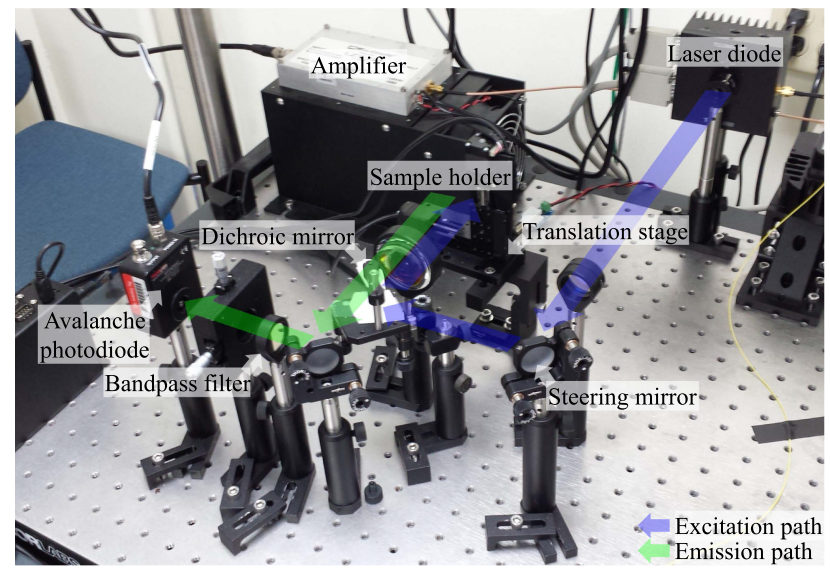

Fig. 13. Hardware prototype. Temporally modulated light from the laser diode travels along the excitation path (in blue) and excites the fluorescent sample. The fluorescent emission from the sample travels along the emission path (in green) and is detected by the avalanche photodiode. The final measurements are obtained by correlation of the emission signal with the demodulation signal from the function generator. The fluorescence lifetime is measured from the final measurements.

galvo-mirrors. In this article, we compare different coding schemes in terms of the SNR or acquisition time per pixel. Although the current prototype system results in slow acquisition due to mechanical scanning, it serves as a test-bed for evaluating relative coding scheme performance. An important next step, although outside the scope of this article, is to implement the proposed coding schemes on optimized hardware for faster and low-cost FLIM estimation.

Details of Hardware Setup. Our setup consists of a laser diode (L450P1600MM, Thorlabs) of wavelength $450 \mathrm{~nm}$ for illuminating the sample of interest. The intensity of the laser diode is modulated by a Siglent SDG 5162 arbitrary function generator for generating arbitrary modulation functions. The function generator has two channels: One is used for generating the modulation functions, and the other for generating the demodulation functions. The modulation signal from the function generator is amplified by the power amplifier with $45 \mathrm{~dB}$ gain (LZY-22+, Mini-Circuits) before applying to the laser diode. For fair comparisons, we used the same average source power and acquisition time for all coding schemes.

The light emitted by the laser diode is reflected by a dichroic mirror and excites a fluorescent sample. A dichroic mirror, which reflects the light below the cutoff wavelength and transmits the light above it, is used to separate the emission signal from the excitation signal. The fluorescent sample is mounted on a translation stage and can move along $\mathrm{x}$ and $\mathrm{y}$ directions for scanning purposes. The translation stage is driven by a stepper controller 
Table 5. Estimated Optimal Parameter Sets

\begin{tabular}{cccc}
\hline & Sinusoid-Sinusoid & Delta-Square & Expo-Square \\
\hline Coumarin 6 in ethanol & $\left\{f_{0}=41.2 \mathrm{MHz}\right\}$ & $\left\{f_{0}=40.0 \mathrm{MHz}, \chi=0.4\right\}$ & $\left\{f_{0}=8.6 \mathrm{MHz}, \kappa=0.55 e 9 \mathrm{~s}^{-1}, \chi=0.34\right\}$ \\
Rhodamine 110 in ethanol & $\left\{f_{0}=26.4 \mathrm{MHz}\right\}$ & $\left\{f_{0}=33.3 \mathrm{MHz}, \chi=0.4\right\}$ & $\left\{f_{0}=5.6 \mathrm{MHz}, \kappa=0.35 e 9 \mathrm{~s}^{-1}, \chi=0.34\right\}$ \\
Fluorescein in ethanol & $\left\{f_{0}=24.4 \mathrm{MHz}\right\}$ & $\left\{f_{0}=30.4 \mathrm{MHz}, \chi=0.4\right\}$ & $\left\{f_{0}=5.1 \mathrm{MHz}, \kappa=0.32 e 9 \mathrm{~s}^{-1}, \chi=0.34\right\}$ \\
\hline
\end{tabular}

The optimal parameter sets of three coding schemes, for three different solutions, were computed using our surrogate.

(MMC-203, Micronix USA). The emission light from the fluorescent sample passes through a bandpass filter (FB550-40, Thorlabs), gets focused by a lens, and then detected by an avalanche photodiode (APD430A2/M, Thorlabs). The bandpass filter is necessary to block the excitation light (wavelength $(\lambda)=450 \mathrm{~nm}$ ) and to allow only the emission light $(\lambda \approx 550 \mathrm{~nm})$. The avalanche photodiode has a high responsivity around $600 \mathrm{~nm}$.

The emission signal detected at the APD is multiplied by the demodulation signal from the function generator using a frequency mixer (ZX05-1L-S+, Mini-Circuits). The multiplied signal from the frequency mixer is integrated using a low-pass filter with a $1 \mathrm{kHz}$ passband (EF110, Thorlabs), digitized by a DAQ (USB-6000, National Instruments) and then recorded by a computer. Note that the demodulation process (computing the correlation of the fluorescence emission with a demodulation signal) is performed electronically in our prototype. In practice, the demodulation can be performed with an image intensifier or lock-in amplifiers (Philip and Carlsson 2003), in full-frame FD-FLIM systems. Figure 13 shows the path taken by light from the source to the sample (the excitation path) and from the sample to the sensor (the emission path).

\subsection{Experimental Results}

8.2.1 Single Pixel Experiment. We measured the lifetimes of three fluorescent samples: Coumarin 6 in ethanol, Rhodamine 110 in ethanol, and Fluorescein in ethanol with the coding schemes used for simulation: Sinusoid-Sinusoid, Delta-Square, and Expo-Square. The concentrations of all three solutions were $0.1 \mathrm{mM}$. The lifetimes of Coumarin 6 in ethanol, Rhodamine 110 in ethanol, and Fluorescein in ethanol are known as $2.5 \mathrm{~ns}, 3.9 \mathrm{~ns}$, and $4.25 \mathrm{~ns}$, respectively (Magde et al. 1999; Shaner et al. 2013; Wilkerson Jr. et al. 1993).

Computing Optimal Parameters. The optimal parameter sets $\mathcal{P}_{\text {opt }}$ of three coding schemes, for each of the three solutions, were computed using our surrogate. We used known lifetime values for the surrogate computation, but, in general, we can use the approximate lifetime range. The computed optimal parameter sets of three coding schemes, for three solutions, are in Table 5. The data acquisition time for each measurement was $10 \mathrm{~ms}$, and a $174 \mathrm{~mA}$ DC signal was used to drive the laser diode for all tested coding schemes for the same average source power constraint. The waveforms were band-limited since the maximum output frequency of the function generator in our setup is limited to $160 \mathrm{MHz}$.

Results: We measured the lifetimes at a single position for each sample 1,000 times, and compared the mean absolute errors of the repeated measurements for the three coding schemes. Figure 14 shows the histograms and the mean absolute errors of the measured lifetimes. For all solutions, Expo-Square achieves approximately three times better performance as compared to
Sinusoid-Sinusoid in terms of the mean absolute error of the measured lifetimes, which is consistent with the surrogate prediction.

8.2.2 Scanning Experiments. In order to compare the performance of different schemes over full-frame fluorescence lifetime images, we created six solutions with different lifetimes by mixing Coumarin 6 and Fluorescein in ethanol in different proportions in cuvettes. The concentration of Coumarin 6 varies linearly from $47.8 \mu \mathrm{M}$ to $13.7 \mu \mathrm{M}$, while that of Fluorescein varies linearly from $0.0 \mu \mathrm{M}$ to $11.9 \mu \mathrm{M}$. Each solution in a cuvette was scanned with our system to get a $50 \times 50$ lifetime image. We compared two coding schemes, Sinusoid-Sinusoid and Expo-Square. $\mathcal{P}_{\text {opt }}=\left\{f_{0}=\right.$ $30.6 \mathrm{MHz}\}$ and $\mathcal{P}_{\text {opt }}=\left\{f_{0}=6.8 \mathrm{MHz}, a=0.4 e 9 \mathrm{~s}^{-1}, \chi=0.34\right\}$ for Sinusoid-Sinusoid and Expo-Square, respectively, were computed using the surrogate based on the lifetime range [2.5ns, $3.9 \mathrm{~ns}]$. The data acquisition time for each pixel was $12.5 \mathrm{~ms}$, and we used the same $168 \mathrm{~mA}$ DC signal to drive the laser diode for two coding schemes to ensure the same average source power.

Figure 15(a) shows the fluorescence lifetime images obtained by Sinusoid-Sinusoid (upper) and Expo-Square (lower). Six $50 \times 50$ lifetime images for the six solutions were combined to form a $300 \times 50$ image. Expo-Square shows more uniform lifetime estimation results than Sinusoid-Sinusoid, whose lifetime image suffers from strong noise. Figure 15(b) shows the mean lifetime $m_{\tau}$ and the mean absolute errors, MAE, of the measured lifetimes for six solutions. $m_{\tau}$ increases as the relative proportion of Fluorescein increases and that of Coumarin 6 decreases.

The measurement mean absolute error, MAE, for SinusoidSinusoid is significantly higher than that for Expo-Square. As a result, the error bars for Sinusoid-Sinusoid overlap across cuvettes, thus making it challenging to differentiate materials with similar lifetimes (neighboring images). This is demonstrated by performing an edge detection on the composite lifetime images for both schemes, as shown in Figure 15(c). For Sinusoid-Sinusoid, many spurious edges are detected within each individual sub-image, in addition to the desired boundary between the solutions. This is because the variation in the estimated lifetimes within each image is large, making it difficult to classify the solutions based on the estimated lifetimes. For Expo-Square, no clear edges are found except the boundaries between the solutions. Therefore, the solutions can be classified well according to the estimated lifetimes.

Figure 1(d) and Figure 16(a) show FLIM results on fluorescent objects made with fluorescent tape and a fluorescent slide such that each object has different lifetimes for the foreground (fluorescent tape) and the background (fluorescent slide). The lifetimes were measured by Sinusoid-Sinusoid and Expo-Square. The data acquisition time for each pixel of the star (Figure 1(d)) and the bird (Figure 16(a) upper) samples was $0.8 \mathrm{~ms}$, while that for the logo sample (Figure 16(a) lower) was $1 \mathrm{~ms}$. We used very 


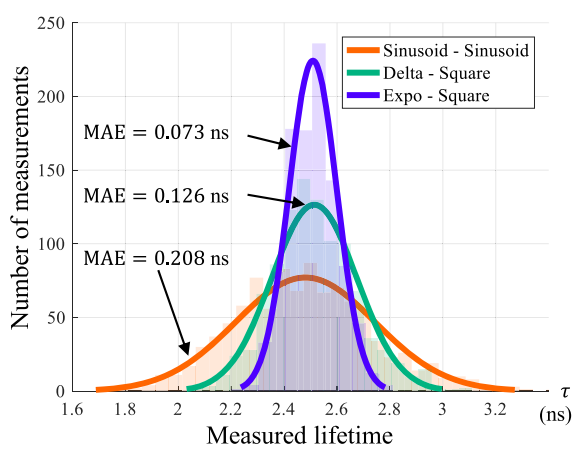

(a) Coumarin 6 in ethanol

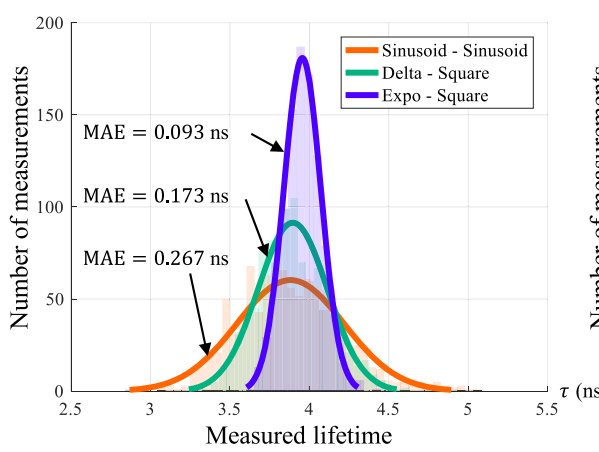

(b) Rhodamine 110 in ethanol

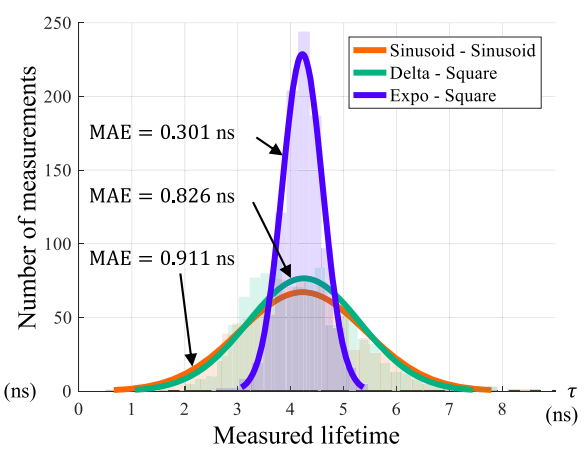

(c) Fluorescein in ethanol

Fig. 14. Single pixel fluorescence lifetime estimation. Lifetimes were measured at single positions for three solutions: (a) Coumarin 6 in ethanol, (b) Rhodamin 110 in ethanol, and (c) Fluorescein in ethanol, using three coding schemes, Sinusoid-Sinusoid, Delta-Square, and Expo-Square. For each solution and each coding scheme, lifetimes were measured 1,000 times, and the histogram and the mean absolute error of the repeated measurements were obtained. Normal distributions were fitted to the histograms for visual comparison. Expo-Square shows approximately three times lower mean absolute error as compared to Sinusoid-Sinusoid, given the same average power and capture time.

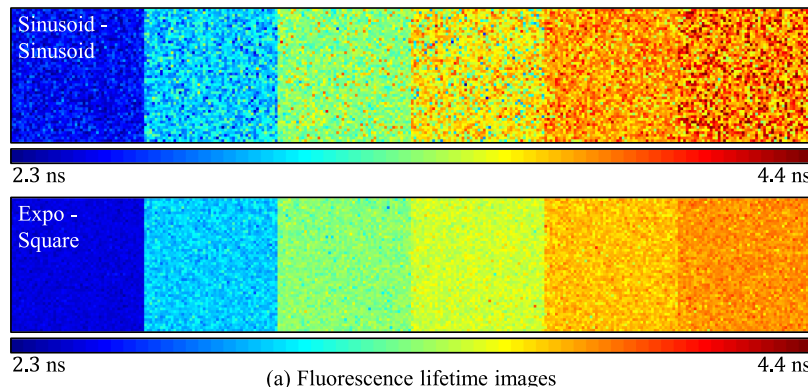

(a) Fluorescence lifetime images

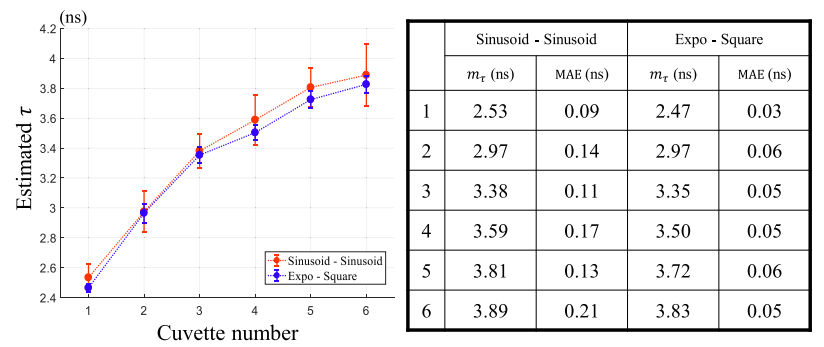

(b) Statistical results

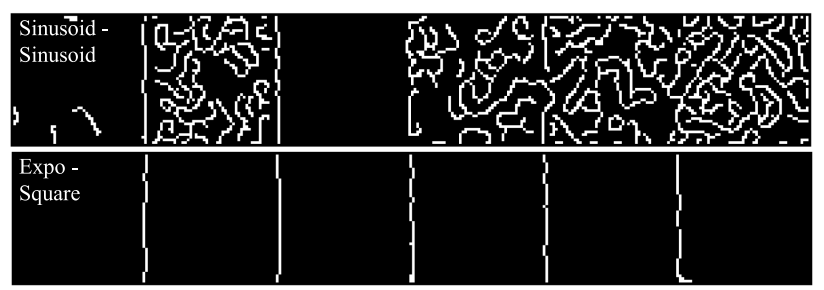

(c) Edge detection results of (a) after smoothing

Fig. 15. Scanning experimental results with solutions. (a) Fluorescence lifetime images of six solutions obtained by Sinusoid-Sinusoid (upper) and Expo-Square (lower). (b) Mean values $m_{\tau}$ and mean absolute errors MAE of the measured lifetimes for six solutions. (c) Edge detection results of (a) after applying the same amount of smoothing to both images. Expo-Square achieves considerably higher SNR as compared to SinusoidSinusoid, resulting in robust determination of edges across the solution boundaries.
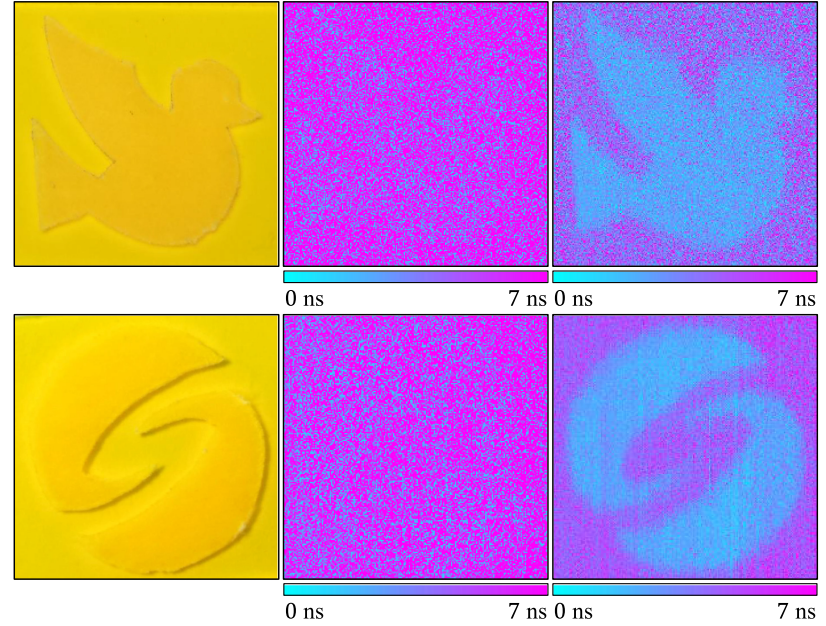

(a) Fluorescent sample

(b) Sinusoid - Sinusoid

(c) Expo - Square

Fig. 16. Visual comparisons of fluorescence lifetime images. (a) Solid fluorescent samples with two lifetimes were made using the fluorescent tape (foreground) and the fluorescent slide (background). The fluorescence lifetime images were obtained by two coding schemes, (b) SinusoidSinusoid and (c) Expo-Square. The boundaries between the foreground and background are invisible with Sinusoid-Sinusoid but clear with ExpoSquare.

low light source power $(151 \mathrm{~mA}$ from the laser diode current controller for the star and the bird samples and $155 \mathrm{~mA}$ for the logo sample), for both coding schemes. The optimal parameter sets determined for these scenes were $\mathcal{P}_{o p t}=\left\{f_{0}=32.5 \mathrm{MHz}\right\}$ and $\mathcal{P}_{\text {opt }}=\left\{f_{0}=6.8 \mathrm{MHz}, a=0.44 \mathrm{e}^{\mathrm{s}} \mathrm{s}^{-1}, \chi=0.34\right\}$ for SinusoidSinusoid and Expo-Square, respectively. The boundaries between the foreground and the background are nearly invisible with Sinusoid-Sinusoid (Figure 1(e) and Figure 16(b)) but clearly discernible with Expo-Square (Figure 1(g) and Figure 16(c)).

8.2.3 Comparison of Data Acquisition Times. We compare the data acquisition times of various schemes, in order to achieve a fixed desired lifetime precision. Figure 17(a) compares the data 


\begin{tabular}{|c|c|c|c|}
\hline \multirow{2}{*}{ Schemes } & \multicolumn{3}{|c|}{ Data acquisition time (ms) } \\
\cline { 2 - 4 } & $\begin{array}{c}\text { Coumarin 6 } \\
\text { in ethanol }\end{array}$ & $\begin{array}{c}\text { Rhodamine 110 } \\
\text { in ethanol }\end{array}$ & $\begin{array}{c}\text { Fluorescein } \\
\text { in ethanol }\end{array}$ \\
\hline Sinusoid - Sinusoid & 10.00 & 10.00 & 10.00 \\
\hline Delta - Square & 3.90 & 3.48 & 8.08 \\
\hline Expo - Square & 1.60 & 1.45 & 1.48 \\
\hline
\end{tabular}

(a) Data acquisition time comparison

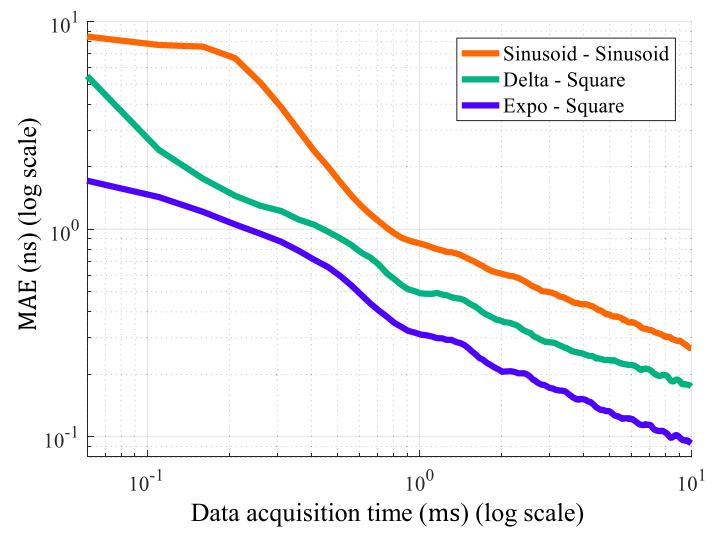

(b) Mean absolute error over the data acquisition time

Fig. 17. Data acquisition time and lifetime precision comparison. (a) Data acquisition time to achieve the same lifetime precision. ExpoSquare can achieve the same precision as Sinusoid-Sinusoid with only oneseventh data acquisition time. (b) Relationship between the mean absolute error, MAE, of the measured lifetimes for Rhodamine 110 in ethanol with data acquisition time. Expo-Square requires considerably shorter acquisition time than Sinusoid-Sinusoid to achieve the same SNR.

acquisition times between three coding schemes (SinusoidSinusoid, Delta-Square, and Expo-Square) to achieve the same lifetime precision (mean absolute errors) for three solutions (Coumarin 6, Rhodamine 110, and Fluorescein in ethanol). The desired mean absolute error was fixed to the mean absolute error achieved by the Sinusoid-Sinusoid with $10 \mathrm{~ms}$ data acquisition time: $0.26 \mathrm{~ns}, 0.34 \mathrm{~ns}$, and $1.19 \mathrm{~ns}$ for Coumarin 6, Rhodamine 110 , and Fluorescein, respectively. Expo-Square can achieve the same precision as Sinusoid-Sinusoid with only one-seventh data acquisition time. Figure 17(b) shows the relationship between the lifetime precision and the data acquisition time. The mean absolute errors, MAE, of the measured lifetimes of Rhodamine 110 in ethanol were plotted against data acquisition time for three coding schemes. Expo-Square achieves high precision (low mean absolute error) even at very short acquisition times, thus leading to considerable speed-ups in data capture, given a desired level of SNR.

\section{LIMITATIONS AND FUTURE WORK}

Objective for Lifetime Precision for a Multi-Exponential Decay. The mean lifetime error and the surrogate proposed in the article are intended for mono-exponential decay (single lifetime). However, for certain materials, the fluorescence emission profile is a linear combination of multiple exponential functions (Lakowicz 2006). An important next step is to extend the approaches developed in this article, and design an objective for lifetime precision, and code optimization procedures for materials with multi-exponential fluorescence lifetime decay.

Surrogate for More Than Three Measurements. The surrogate, as defined in Equation (16), is valid only for $K=3$ measurements since the cross product in Equation (13) is not generally defined when $K>3$. While this is not a strong practical limitation since the relative performance of two coding schemes remains approximately the same irrespective of $K$, a promising direction of future research is to derive a surrogate for $K>3$ measurements.

Optimization of the Surrogate. The proposed surrogate as an objective function is non-convex and non-smooth. Hence, it is challenging to obtain globally optimal solutions for both modulation and demodulation functions in the general case (i.e., when the search space contains all possible coding schemes). A compelling line of research is to seek potentially sub-optimal solutions in a narrowed search space by incorporating various physical constraints like the maximum peak power and the maximum system bandwidth.

\section{ACKNOWLEDGMENTS}

The authors would also like to thank Felipe Gutierrez Barragan for calibration of the setup.

\section{REFERENCES}

Diego Airado-Rodríguez, Teresa Galeano-Díaz, Isabel Durán-Merás, and Jens Petter Wold. 2009. Usefulness of fluorescence excitation-emission matrices in combination with PARAFAC, as fingerprints of red wines. Fournal of Agricultural and Food Chemistry 57, 5 (2009), 1711-1720.

Syed Abdullah Aljunid, Gleb Maslennikov, Yimin Wang, Hoang Lan Dao, Valerio Scarani, and Christian Kurtsiefer. 2013. Excitation of a single atom with exponentially rising light pulses. Physical Review Letters 111, 10 (2013), 103001.

Philippe I. H. Bastiaens and Anthony Squire. 1999. Fluorescence lifetime imaging microscopy: Spatial resolution of biochemical processes in the cell. Trends in Cell Biology 9, 2 (1999), 48-52.

Wolfgang Becker. 2014. The bh TCSPC Handbook. Becker \& Hickl.

Oscar Beijbom, Tali Treibitz, David I. Kline, Gal Eyal, Adi Khen, Benjamin Neal, Yossi Loya, B. Greg Mitchell, and David Kriegman. 2016. Improving automated annotation of benthic survey images using wide-band fluorescence. Scientific Reports 6 (2016), 23166.

Mikhail Y. Berezin and Samuel Achilefu. 2010. Fluorescence lifetime measurements and biological imaging. Chemical Reviews 110, 5 (2010), 2641-2684.

Ayush Bhandari, Christopher Barsi, and Ramesh Raskar. 2015a. Blind and referencefree fluorescence lifetime estimation via consumer time-of-flight sensors. Optica 2, 11 (Nov. 2015), 965-973. DOI : https://doi.org/10.1364/OPTICA.2.000965

Ayush Bhandari, Christopher Barsi, and Ramesh Raskar. 2015b. Blind and referencefree fluorescence lifetime estimation via consumer time-of-flight sensors. Optica 2, 11 (2015), 965-973.

Ayush Bhandari and Ramesh Raskar. 2016. Signal processing for time-of-flight imaging sensors: An introduction to inverse problems in computational 3-D imaging. IEEE Signal Processing Magazine 33, 5 (2016), 45-58.

M. J. Booth and T. Wilson. 2004. Low-cost, frequency-domain, fluorescence lifetime confocal microscopy. Journal of Microscopy 214, 1 (2004), 36-42.

A. Colasanti, A. Kisslinger, G. Fabbrocini, R. Liuzzi, M. Quarto, P. Riccio, G. Roberti, and F. Villani. 2000. MS-2 fibrosarcoma characterization by laser induced autofluorescence. Lasers in Surgery and Medicine 26, 5 (2000), 441-448.

Daniela Comelli, Cosimo D'Andrea, Gianluca Valentini, Rinaldo Cubeddu, Chiara Colombo, and Lucia Toniolo. 2004. Fluorescence lifetime imaging and spectroscopy as tools for nondestructive analysis of works of art. Applied Optics 43, 10 (2004), 2175-2183.

Kalyanmoy Deb, Amrit Pratap, Sameer Agarwal, and TAMT Meyarivan. 2002. A fast and elitist multiobjective genetic algorithm: NSGA-II. IEEE Transactions on Evolutionary Computation 6, 2 (2002), 182-197.

Michelle A. Digman, Valeria R. Caiolfa, Moreno Zamai, and Enrico Gratton. 2008. The phasor approach to fluorescence lifetime imaging analysis. Biophysical fournal 94, 2 (2008), L14-L16.

L. A. Donaldson and K. Radotic. 2013. Fluorescence lifetime imaging of lignin autofluorescence in normal and compression wood. fournal of Microscopy 251, 2 (2013), $178-187$. 
Alan Elder, Simon Schlachter, and Clemens F. Kaminski. 2008. Theoretical investigation of the photon efficiency in frequency-domain fluorescence lifetime imaging microscopy. FOSA A 25, 2 (2008), 452-462.

Dan Elson, Jose Requejo-Isidro, Ian Munro, Fred Reavell, Jan Siegel, Klaus Suhling, Paul Tadrous, Richard Benninger, Peter Lanigan, James McGinty, et al. 2004. Timedomain fluorescence lifetime imaging applied to biological tissue. Photochemical \& Photobiological Sciences 3, 8 (2004), 795-801.

Alessandro Esposito, Hans Gerritsen, Thierry Oggier, Felix Lustenberger, and Fred S. Wouters. 2006. Innovating lifetime microscopy: A compact and simple tool for life sciences, screening, and diagnostics. Journal of Biomedical Optics 11, 3 (2006), 034016.

Alessandro Esposito, Hans C. Gerritsen, and Fred S. Wouters. 2007. Optimizing frequency-domain fluorescence lifetime sensing for high-throughput applications: Photon economy and acquisition speed. FOSA A 24, 10 (2007), 3261-3273.

A. Esposito, T. Oggier, H. C. Gerritsen, F. Lustenberger, and F. S. Wouters. 2005. Allsolid-state lock-in imaging for wide-field fluorescence lifetime sensing. Optics Ex press 13, 24 (2005), 9812-9821.

Ying Fu, Antony Lam, Yasuyuki Matsushita, Imari Sato, and Yoichi Sato. 2014. Interreflection removal using fluorescence. In European Conference on Computer Vision. Springer, 203-217.

Mohit Gupta, Andreas Velten, Shree K. Nayar, and Eric Breitbach. 2018. What are optimal coding functions for time-of-flight imaging? ACM Transactions on Graphics (TOG) 37, 2 (2018), 13.

Shuai Han, Yasuyuki Matsushita, Imari Sato, Takahiro Okabe, and Yoichi Sato. 2012. Camera spectral sensitivity estimation from a single image under unknown illumination by using fluorescence. In IEEE Conference on Computer Vision and Pattern Recognition (CVPR'12). IEEE, 805-812.

Miles Hansard, Seungkyu Lee, Ouk Choi, and Radu Patrice Horaud. 2012. Time-ofFlight Cameras: Principles, Methods and Applications. Springer Science \& Business Media.

Samuel W. Hasinoff, Frédo Durand, and William T. Freeman. 2010. Noise-optimal capture for high dynamic range photography. In IEEE Conference on Computer Vision and Pattern Recognition (CVPR'10). IEEE, 553-560.

Felix Heide, Matthias B. Hullin, James Gregson, and Wolfgang Heidrich. 2013. Lowbudget transient imaging using photonic mixer devices. ACM Transactions on Graphics (ToG) 32, 4 (2013), 45

Felix Heide, Lei Xiao, Andreas Kolb, Matthias B. Hullin, and Wolfgang Heidrich. 2014 Imaging in scattering media using correlation image sensors and sparse convolutional coding. Optics Express 22, 21 (2014), 26338-26350.

J. Michael Hollas. 2004. Modern Spectroscopy. John Wiley \& Sons.

Matthias B. Hullin, Martin Fuchs, Ivo Ihrke, Hans-Peter Seidel, and Hendrik P. A. Lensch. 2008. Fluorescent immersion range scanning. ACM Transactions on Graphics 27, 3 (Aug. 2008), Article 87, 10 pages. DOI:https://doi.org/10.1145/ 1360612.1360686

Matthias B. Hullin, Johannes Hanika, Boris Ajdin, Hans-Peter Seidel, Jan Kautz, and Hendrik P. A. Lensch. 2010. Acquisition and analysis of bispectral bidirectional reflectance and reradiation distribution functions. ACM Transactions on Graphics 29, 4 (July 2010), Article 97, 7 pages. DOI : https://doi.org/10.1145/1778765.1778834

Moon S. Kim, Byoung-Kwan Cho, Alan M. Lefcourt, Yud-Ren Chen, and Sukwon Kang. 2008. Multispectral fluorescence lifetime imaging of feces-contaminated apples by time-resolved laser-induced fluorescence imaging system with tunable excitation wavelengths. Applied Optics 47, 10 (2008), 1608-1616.

Joseph R. Lakowicz. 2006. Principles of Fluorescence Spectroscopy. Springer

R. Lange. 2000. 3D Time of Flight Distance Measurement with Custom Solid State Image Sensors in CMOS, CCD Technology. https://books.google.com/books?id= upMuHwAACAAJ

Lea Lenhardt, Rasmus Bro, Ivana Zeković, Tatjana Dramićanin, and Miroslav D Dramićanin. 2015. Fluorescence spectroscopy coupled with PARAFAC and PLS DA for characterization and classification of honey. Food Chemistry 175 (2015), 284-291.

Yuxiang Lin and Arthur F. Gmitro. 2010. Statistical analysis and optimization of frequency-domain fluorescence lifetime imaging microscopy using homodyne lock-in detection. 7OSA A 27, 5 (2010), 1145-1155.

Douglas Magde, Gail E. Rojas, and Paul G. Seybold. 1999. Solvent dependence of the fluorescence lifetimes of xanthene dyes. Photochemistry and Photobiology 70, 5 (1999), 737-744.
John A. Nelder and Roger Mead. 1965. A simplex method for function minimization. The Computer fournal 7, 4 (1965), 308-313.

Austin Nevin, Daniela Comelli, Gianluca Valentini, Demetrios Anglos, Aviva Burnstock, Sharon Cather, and Rinaldo Cubeddu. 2007. Time-resolved fluorescence spectroscopy and imaging of proteinaceous binders used in paintings. Analytical and Bioanalytical Chemistry 388, 8 (2007), 1897-1905.

Lucio Pancheri, Nicola Massari, and David Stoppa. 2013. SPAD image sensor with analog counting pixel for time-resolved fluorescence detection. IEEE Transactions on Electron Devices 60, 10 (2013), 3442-3449.

Johan Philip and Kjell Carlsson. 2003. Theoretical investigation of the signal-to-noise ratio in fluorescence lifetime imaging. FOSA A 20, 2 (2003), 368-379.

Asima Pradhan, Prabir Pal, Gilles Durocher, Luc Villeneuve, Antonia Balassy, Féridoun Babai, Louis Gaboury, and Louise Blanchard. 1995. Steady state and time-resolved fluorescence properties of metastatic and non-metastatic malignant cells from different species. Fournal of Photochemistry and Photobiology B: Biology 31, 3 (1995), 101-112.

J. Requejo-Isidro, J. McGinty, I. Munro, D. S. Elson, N. P. Galletly, M. J. Lever, M. A A. Neil, G. W. H. Stamp, P. M. W. French, P. A. Kellett, et al. 2004. High-speed wide-field time-gated endoscopic fluorescence-lifetime imaging. Optics Letters 29 19 (2004), 2249-2251.

Imari Sato, Takahiro Okabe, and Yoichi Sato. 2012. Bispectral photometric stereo based on fluorescence. In IEEE Conference on Computer Vision and Pattern Recognition (CVPR'12). IEEE, 270-277.

S. Schlachter, A. D. Elder, A. Esposito, G. S. Kaminski, J. H. Frank, L. K. Van Geest, and C. F. Kaminski. 2009. mhFLIM: Resolution of heterogeneous fluorescence decays in widefield lifetime microscopy. Optics Express 17, 3 (2009), 1557-1570.

L. K. Seah, U. S. Dinish, W. F. Phang, Z. X. Chao, and V. M. Murukeshan. 2005. Fluorescence optimisation and lifetime studies of fingerprints treated with magnetic powders. Forensic Science International 152, 2-3 (2005), 249-257.

L. K. Seah, P. Wang, V. M. Murukeshan, and Z. X. Chao. 2006. Application of fluorescence lifetime imaging (FLIM) in latent finger mark detection. Forensic Science International 160, 2-3 (2006), 109-114.

Nathan C. Shaner, Gerard G. Lambert, Andrew Chammas, Yuhui Ni, Paula J. Cranfill, Michelle A. Baird, Brittney R. Sell, John R. Allen, Richard N. Day, Maria Israelsson, et al. 2013. A bright monomeric green fluorescent protein derived from Branchiostoma lanceolatum. Nature Methods 10, 5 (2013), 407.

Wen Shi, Xiaohua Li, and Huimin Ma. 2014. Fluorescent probes and nanoparticles for intracellular sensing of $\mathrm{pH}$ values. Methods and Applications in Fluorescence 2, 4 (2014), 042001.

Ewa Sikorska, Tomasz Górecki, Igor V. Khmelinskii, Marek Sikorski, and Jacek Kozioł 2005. Classification of edible oils using synchronous scanning fluorescence spectroscopy. Food Chemistry 89, 2 (2005), 217-225.

Shiwen Sun, Birgit Ungerböck, and Torsten Mayr. 2015. Imaging of oxygen in microreactors and microfluidic systems. Methods and Applications in Fluorescence 3 3 (2015), 034002

Virginia Torczon. 1997. On the convergence of pattern search algorithms. SIAM four nal on Optimization 7, 1 (1997), 1-25.

Tali Treibitz, Zak Murez, B. Greg Mitchell, and David Kriegman. 2012. Shape from fluorescence. In European Conference on Computer Vision. Springer, 292-306.

Andreas Velten, Thomas Willwacher, Otkrist Gupta, Ashok Veeraraghavan, Moung G. Bawendi, and Ramesh Raskar. 2012. Recovering three-dimensional shape around a corner using ultrafast time-of-flight imaging. Nature Communications 3 (2012), 745.

Charles W. Wilkerson Jr., Peter M. Goodwin, W. Patrick Ambrose, John C. Martin, and Richard A. Keller. 1993. Detection and lifetime measurement of single molecules in flowing sample streams by laser-induced fluorescence. Applied Physics Letters 62, 17 (1993), 2030-2032

Di Wu, Matthew O'Toole, Andreas Velten, Amit Agrawal, and Ramesh Raskar. 2012 Decomposing global light transport using time of flight imaging. In IEEE Conference on Computer Vision and Pattern Recognition (CVPR'12). IEEE, 366-373.

Qiaole Zhao, Ian T. Young, and Jan Geert Sander De Jong. 2011. Photon budget analysis for fluorescence lifetime imaging microscopy. Fournal of Biomedical Optics 16, 8 (2011), 086007.

Received June 2018; revised November 2018; accepted March 2019 